\title{
Two-loop Higgs mass calculations in supersymmetric models beyond the MSSM with SARAH and SPheno
}

\author{
Mark D. Goodsell ${ }^{1}$, Kilian Nickel $^{2}$, Florian Staub ${ }^{2,3, a}$ \\ ${ }^{1}$ UMR 7589, LPTHE, Sorbonne Universités, UPMC Univ Paris 06, CNRS, 75005 Paris, France \\ ${ }^{2}$ BCTP and Physikalisches Institut der Universität Bonn, Nußallee 12, 53115 Bonn, Germany \\ ${ }^{3}$ Theory Division, CERN, 1211 Geneva 23, Switzerland
}

Received: 10 November 2014 / Accepted: 22 December 2014 / Published online: 27 January 2015

(C) The Author(s) 2015. This article is published with open access at Springerlink.com

\begin{abstract}
We present an extension to the Mathematica package SARAH which allows for Higgs mass calculations at the two-loop level in a wide range of supersymmetric (SUSY) models beyond the MSSM. These calculations are based on the effective potential approach and include all two-loop corrections which are independent of electroweak gauge couplings. For the numerical evaluation Fortran code for SPheno is generated by SARAH. This allows the prediction of the Higgs mass in more complicated SUSY models with the same precision that most state-of-the-art spectrum generators provide for the MSSM.
\end{abstract}

\section{Introduction}

The discovery of the Higgs boson has been so far the biggest success of the experiments at the Large Hadron Collider (LHC) $[1,2]$. The mass of the Higgs is already pinned down with an impressive experimental uncertainty of just a few hundred $\mathrm{MeV}$ in the range of $125-126 \mathrm{GeV}$. This experimental accuracy is at the moment much better than theoretical predictions for the Higgs mass in any given model beyond the standard model (SM). For instance, in recent decades a lot of effort has been taken to calculate the Higgs mass in the minimal supersymmetric standard model (MSSM). This industry was initiated by the observation that stop corrections can lift the Higgs mass, which is bounded at treelevel to be below $M_{Z}$, above the long existing LEP limit of $114 \mathrm{GeV}$ [3-7]. The next milestones were a complete diagrammatic one-loop calculation [8-11] and a calculation of the leading two-loop corrections in the effective potential approach [12-26] or equivalent diagrammatic calcula-

BONN-TH-2014-14, CERN-PH-TH-2014-213.

a e-mail: florian.staub@cern.ch tions with zero external momentum [27] on the one side as well as progressively better calculations using renormalisation group equation (RGE) methods [28-34]. In particular the two-loop calculation in the effective potential including $O\left(\alpha_{s}\left(\alpha_{t}+\alpha_{b}+\alpha_{\tau}\right)\right)$ and $O\left(\left(\alpha_{t}+\alpha_{b}+\alpha_{\tau}\right)^{2}\right)$ corrections are widely used because they have entered different public codes such as SoftSUSY [35-37], SPheno [38,39], Suspect [40] or FeynHiggs [41,42]. The RGE methods are implemented in CPsuperH $[43,44]$. Also three-loop results in the effective potential approach exist [45-47]. The discovery of the Higgs and the determination of its mass gave a new impetus to these calculations and now the diagrammatic two-loop calculations $O\left(\alpha_{s} \alpha_{t}\right)$ and $O\left(\alpha_{t}^{2}\right)$ including external momenta exist [48-51]. In addition, calculations in an effective model matched to the MSSM at a higher scale have been performed [52,53]. However, if one goes beyond the MSSM and considers non-minimal SUSY models, the picture is very simple: only for the next-to-minimal supersymmetric standard model (NMSSM) a full one-loop calculation has been presented in the literature [54-57]. At the two-loop level only the $O\left(\alpha_{s}\left(\alpha_{t}+\alpha_{b}\right)\right)$ in the effective potential approach are known up to now [54]. This is in particular problematic because non-minimal SUSY models have gained more and more interest in the last few years because of the experimental results: (i) they can lift the Higgs mass at tree-level by new $F$ - or $D$-contributions [58-62] which makes these models more natural by reducing the fine-tuning [63-67], (ii) they can weaken SUSY limits by either predicting compressed spectra or reducing the expected missing transverse energy significantly [68-71]. A brief overview of Higgs sectors in BMSSM models is given, for instance, in [72].

The situation in non-minimal SUSY models has been relaxed with the development of the Mathematica package SARAH [73-77]: SARAH can automatically generate SPheno modules which allow for a full one-loop calculation in a wide range of SUSY models like singlet 
extensions $[67,78,79]$, triplet extensions [80], models with Dirac gauginos and broken $[81,82]$ or unbroken [83] $R$ symmetry, extended gauge sectors $[62,84-86]$ or even more exotic models $[87,88]$. A similar link between SARAH and FlexibleSUSY has been presented recently [89]. These automatised calculations have now been brought to the next level by providing routines which calculate two-loop corrections to the $\mathrm{CP}$ even Higgs scalars in the effective potential approach. The resulting accuracy for many beyond-MSSM models is the same as for the MSSM using the results of Refs. [20,21,23,24,26]. For this purpose, the generic results for the two-loop effective potential presented in Ref. [90] have been implemented in SARAH and the two-loop selfenergies in the approximation of vanishing external momenta are calculated using a numerical derivation. This is analog to Ref. [22]. Corrections including gauge couplings of a broken gauge group are not included.

This paper is organized as follows. In Sect. 2 we review the effective potential approach as well as our implementation in SARAH and SPheno. In Sect. 3 we compare the results obtained for the MSSM and NMSSM with the results of well established routines. In Sect. 4 we explain how the user can obtain the two-loop results for his/her favorite model before we conclude in Sect. 5 .

\section{Effective potential approach for two-loop Higgs masses in a generic SUSY model}

We consider a set of real scalars $\left\{\phi_{k}\right\}$ which are diagonalised to physical states $\left\{h_{k}\right\}$. The scalar potential at tree-level is $V^{T}\left(\left\{\phi_{k}\right\}\right)$. The pole masses of $h_{i}$ are in general the eigenvalues of the loop corrected mass matrix given by

$\frac{\partial^{2} V^{T}}{\partial \phi_{i} \partial \phi_{j}}+\sum_{n} \Pi_{i j}^{(n)}\left(p^{2}\right)$

Here, $\Pi^{(n)}\left(p^{2}\right)$ is the self-energy at the $n$-loop level which usually depends on the external momenta. The parameters appearing in $V^{T}$ should be chosen to minimise the effective potential. For a pole mass $m_{h_{i}}$ the momentum is fixed to be $p^{2}=m_{h_{i}}^{2}$. To include this momentum dependence of $\Pi^{(n)}$ a diagrammatic calculation is necessary. However, things become significantly easier if one considers the limit of $p^{2}=0$. In this limit $\Pi^{(n)}(0)$ is equivalent to the second derivative of the effective potential at the $n$-loop level,

$\Pi_{i j}^{(n)}(0)=\frac{\partial^{2} V^{(n)}}{\partial \phi_{i} \partial \phi_{j}}$.

We present here a fully automatised hybrid method implemented in the public tools SARAH and SPheno for the calculation of the scalar masses at the two-loop level in the $\overline{\mathrm{DR}}^{\prime}$ renormalization scheme [91]: while the one-loop corrections are calculated including the full momentum dependence, the two-loop corrections are derived in the effective potential approximation. In general, the setup is based on the following work distribution: the user implements their favourite model in SARAH. SARAH derives all analytical expressions for mass-matrices, vertices, renormalisation group equations as well as loop corrections and exports this information into Fortran source code. The Fortran source code is compiled together with SPheno and all numerical calculations are then performed by the new SPheno module. Since the one-loop diagrammatic calculation has been included since SARAH 2.0 , we focus in the following on the new two-loop corrections which are published in version 4.4 .0 . We start with a discussion how the two-loop self-energies are derived.

\subsection{Calculation of the two-loop self energies in the effective potential approximation}

We shall neglect $\mathrm{CP}$-violating effects in the following. Therefore, a set of neutral, complex scalars $H_{i}$ are decomposed after symmetry breaking as

$H_{i}=\frac{1}{\sqrt{2}}\left(\phi_{i}+i \sigma_{i}+v_{i}\right)$

$\phi_{i}$ are the CP-even components, $\sigma_{i}$ the CP-odd ones and $v_{i}$ are the vacuum expectation values (VEVs). Under this assumption, the two-loop corrections to the mass matrix of real scalars in the effective potential approach are given by

$\Pi_{i j}^{(2)}(0)=\frac{\partial^{2} V^{(2)}}{\partial v_{i} \partial v_{j}}$.

In addition, we require the tadpole contributions which are the first derivatives of the effective potential,

$\delta t_{i}^{(2)}=\frac{\partial V^{(2)}}{\partial v_{i}}$.

The tadpoles then also contribute to the scalar masses by shifting the parameters; for example if we treat the VEVs $v_{i}$ as fixed, "all-loop" correct values, then we can exchange them for scalar mass-squared at a given loop order via the tadpole equations, and in turn find compact equations for the total shift in the mass-squared of

$\left(\Delta \mathcal{M}_{S}^{2}\right)_{i j}^{\text {eff }}=\frac{\partial^{2} V^{(2)}}{\partial v_{i} \partial v_{j}}-\frac{\delta_{i j}}{v_{i}} \frac{\partial V^{(2)}}{\partial v_{i}}$

as used, for example, in [54]. However, we do not do this here, instead (as detailed below) solving the tadpole equations and then using the parameters derived from these in the treelevel mass calculation. The reason is that SARAH allows for a more general choice of variables to solve for via the tadpole equations. 


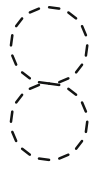

$S S$

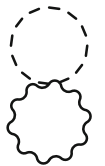

SV

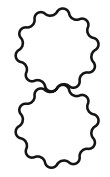

VV

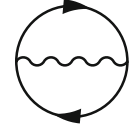

FFV

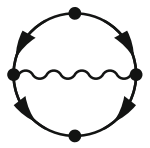

$\overline{F F} V$

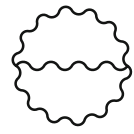

$V V V$

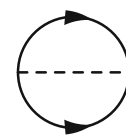

FFS

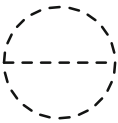

$S S S$

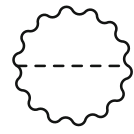

$V V S$

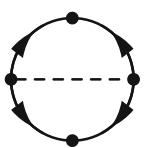

$\overline{F F} S$

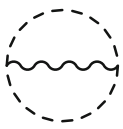

$S S V$

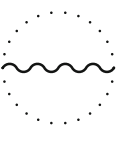

$G G V$
Fig. 1 All possible topologies of two-loop bubble diagrams. We consider in the following $S S, S S V, S S S, F F S, \overline{F F} S, F F V$, and $\overline{F F} V$

$V^{(2)}$ receives contributions from the possible topologies shown in Fig. 1. The generic results for all of these diagrams in Landau gauge have been presented in Ref. [90] and we heavily make use of these results in the following. However, we will not require all topologies but neglect $V V$, $V V S, V V V, S V$, and $G G V$. The reason is that these only lead to non-zero contributions if massive vector bosons are involved. We will neglect all contributions stemming from broken gauge groups because of the many complications that they entail.

The remaining topologies are those which have been considered so far in the MSSM to obtain the dominant two-loop corrections and which are implemented in public computer tools. The impact on the Higgs mass is considered to be moderate when neglecting contributions proportional to the electroweak gauge couplings in models with the SM gauge sector, since they are significantly smaller than the strong and top Yukawa coupling-and the diagrams involving these couplings are of typically lower multiplicity. In fact, by inspecting the form of the loop functions it is evident that the topologies $S V, V V, V V V, V V S$ and $G G V$ lead to contributions proportional to $g_{E W}^{4} v^{2}$ where $g_{E W}$ stands for the electroweak gauge coupling; these are hence subdominant to the contributions from $S S V, F F V, \overline{F F} V$ diagrams involving the electroweak gauge bosons (which we are also neglecting) which would be proportional to $g_{E W}^{2} v^{2}$.

The electroweak contributions in the MSSM have been estimated to be of $O(1 \mathrm{GeV})$ [92]. A more recent estimate of the total theoretical uncertainty of the MSSM Higgs mass concluded that missing two- and three-loop contributions together can account for a shift of about $2 \mathrm{GeV}$ in the Higgs mass if third generation squarks are $2 \mathrm{TeV}$ or lighter [93]. This is much smaller than the corrections involving superpotential interactions and the strong coupling. Of course, in models with extended gauge sectors this conclusion might change. An estimate of the importance of the missing contributions can be obtained be considering the different one-loop corrections and assuming a similar behavior at two loops.

Note, for consistency, we also set all gauge couplings arising in the $D$-terms of broken groups in the vertices to zero. The masses used in the loops are tree-level masses calculated from running $\overline{\mathrm{DR}}^{\prime}$ parameters. In this context, there are two possibilities to treat $D$-term contributions to the tree-level masses: either one can work in the gaugeless limit where these contributions are also put to zero [21], or one can work with the full tree-level masses as this is also done in diagrammatic calculations [49]. We offer both possibilities. In the gaugeless limit the $S U(2)_{L} \times U(1)_{Y}$ gauge symmetries become global symmetries. Therefore, it is obvious that no gauge dependence has been introduced by including only Goldstone diagrams but no diagrams of the corresponding vector bosons. Of course, when including $D$-terms in the mass matrices the derivatives of the $D$-terms are still forced to vanish. However, we stress that the second method has to be used carefully as explained in Sect. 2.3.

For the calculation of the two-loop effective potential we have translated the expressions of Ref. [90] given in two-component notation into four-component language, see Appendix A. All necessary generic expressions have been implemented in SARAH. SARAH uses these expressions to generate Fortran code for all two-loop diagrams which are possible in the considered model assuming the topologies $S S, S S V, S S S, F F S, \overline{F F} S, F F V$ and $\overline{F F V}$.

As soon as the two-loop effective potential is calculated, one can obtain the two-loop corrections to the Higgs mass by performing the derivative of Eqs. (4) and (5) numerically. The numerical derivation in SPheno is done by using Ridders' method of polynomial extrapolation with dynamical stepsize [94]. We have implemented two different methods to take the derivation of the effective potential which the user can choose as explained later in Sect. 4:

1. Purely numerical derivation: in this approach the entire potential is derived with respect to the VEVs. This is the ansatz of Ref. [22].

2. Semi-analytical derivation: in this approach the derivatives of all masses and couplings with respect to the VEVs are calculated separately also in a numerical way. However, all one- and two-loop derivatives of the loopfunctions with respect to their arguments have been calculated analytically and implemented in the output SPheno code. The derivatives of the potential are then 
calculated combining both results using the chain rule. This can be easily done because every contribution to the potential is a product of couplings $\left(c_{1}, c_{2}\right)$, masses $\left(m_{i}\right)$ and a loop function $f_{X}$ with a coefficient $k$, Eq. (7).

$$
\begin{aligned}
V_{X}^{(2)}= & k \cdot\left(c_{1} c_{2}\right) \cdot f_{X}\left(m_{1}^{2}, m_{2}^{2}, m_{3}^{2}\right), \\
& \text { for } X=F F S, F F V, S S V \\
V_{X}^{(2)}= & k \cdot\left(c_{1} c_{2}\right) \cdot m_{F 1} m_{F 2} \cdot f_{X}\left(m_{F 1}^{2}, m_{F 2}^{2}, m_{3}^{2}\right), \\
& \text { for } X=\overline{F F} S, \overline{F F} V \\
V_{X}^{(2)}= & k \cdot\left(c_{1}\right) \cdot f_{X}\left(m_{1}^{2}, m_{2}^{2}\right), \quad \text { for } X=S S
\end{aligned}
$$

The second method is numerically slightly more expensive but it is also more stable. In particular, in the presence of large hierarchies in the VEVs the purely numerical method could become inaccurate. More details about the numerical stability are given in Sect. 4. There is in addition, a third, fully analytical method-which gives results equivalent to a diagrammatic calculation of the pole mass with external momenta set to zero. We will present the analytic results and implementation in a forthcoming publication [95].

\subsection{Calculation of loop corrected mass spectrum}

We have described how the corrections to the effective potential at the two-loop level are calculated and how to obtain the self-energies of the Higgs from it. We will now show how this fits into the full picture by explaining the different steps performed in the numerical evaluation by SPheno to obtain the loop corrected Higgs masses:

1. The starting point for all loop calculations is the set of running parameters at the renormalization scale $Q$. This scale can be either be a fixed value or a variable which depends on other parameters of the model. For instance, in SUSY models it is common to choose $Q$ to be the geometric mean of the stop masses.

2. The running parameters are used to solve the minimisation conditions of the vacuum (the tadpole equations $\left.T_{i}\right)$ at tree-level

$T_{i}=\frac{\partial V^{(T)}}{\partial v_{i}} \equiv 0$.

These equations are solved for a set of parameters, one per equation. This set is determined by the user; typically these are mass-squared parameters, which can be solved for linearly, but SARAH also allows non-linear tadpole equations.

3. The running parameters as well as the solutions of the tadpole equations are used to calculate the tree-level mass spectrum. The tree-level Higgs masses $m_{i}^{h,(T)}$ are the eigenvalues of the tree-level mass matrix $M^{h,(T)}$ defined by

$M^{h,(T)}=\frac{\partial^{2} V^{(T)}}{\partial \phi_{i} \partial \phi_{j}}$

4. Similarly, the tree-level masses of all other particles present in the model are calculated.

5. Using the tree-level masses the one-loop corrections $\delta M_{Z}$ to the $Z$ boson are calculated.

6. The electroweak VEV $v$ is expressed by the measured pole mass of the $Z, M_{Z}^{\text {pole }}$, the one-loop corrections and a function of the involved gauge couplings $g_{i}$.

$v=\sqrt{\frac{M_{Z}^{2, \text { pole }}+\delta M_{Z}^{2}}{f\left(\left\{g_{i}\right\}\right)}}$

In the case of the MSSM $f\left(\left\{g_{i}\right\}\right)=f\left(g_{1}, g_{2}\right)=\frac{1}{4}\left(g_{1}^{2}+\right.$ $g_{2}^{2}$ ) holds. Together with the value of the running $\tan \beta$, the values for the VEVs of the up- and down Higgs can be calculated.

7. The tree-level masses are calculated again with the new values for the VEVs.

8. The one- $\left(\delta t_{i}^{(1)}\right)$ and two-loop $\left(\delta t_{i}^{(2)}\right)$ corrections to the tadpole equations are calculated. These are used to solve the loop-corrected minimisation conditions

$T_{i}+\delta t_{i}^{(1)}+\delta t_{i}^{(2)} \equiv 0$.

9. The one-loop self-energies for all particles including the external momentum $p$ are calculated. For the Higgs, we call them in the following $\Pi^{h,(1 L)}\left(p^{2}\right)$.

10. For the CP-even Higgs states, the two-loop self-energies (with zero external momentum) $\Pi^{h,(2 L)}(0)$ are calculated as explained in the previous section.

11. The physical Higgs masses are then calculated by taking the real part of the poles of the corresponding propagator matrices

$\operatorname{Det}\left[p_{i}^{2} \mathbf{1}-M^{h,(2 L)}\left(p^{2}\right)\right]=0$,

where

$M^{2,(2 L)}\left(p^{2}\right)=\tilde{M}^{h,(T)}-\Pi^{h,(1 L)}\left(p^{2}\right)-\Pi^{h,(2 L)}(0)$.

Here, $\tilde{M}^{h,(T)}$ is the tree-level mass matrix where the parameters solving the loop-corrected tadpole equations are used. Eq. (12) is solved for each eigenvalue $p^{2}=m_{i}^{2}$ in an iterative way. 


\subsection{Treating the Goldstones}

It is well known that the derivatives of the effective potential in the Landau gauge may suffer from divergences due to massless Goldstone bosons [96]: the derivatives of some loop functions have infra-red singularities. In the gaugeless limit of the MSSM, this problem is circumvented because the masses of the pseudoscalars become independent of the Higgs VEVs, and so the derivatives of the effective potential do not contain any singular functions. However, once we go beyond the MSSM (even to the NMSSM) this problem reappears. The singularities in the first derivative of the potential may be tamed by resummation methods [97,98], but the second derivatives may remain singular and it has been suggested that the problem could be resolved by passing to the pole mass calculation instead [98]. However, working in the gaugeless limit this problem is usually not present: as stated above, the tree-level masses entering the calculation are calculated at the minimum of (full) tree-level potential. Thus, using the Lagrangian parameters at this minimum in the mass matrices expressed in the gaugeless limit, the Goldstone masses are usually non-zero and no divergences in the numerical evaluation show up. This is one strong motivation for working in the gaugeless limit: it reduces the dependence of the tree-level Goldstone "masses" that enter into the calculation on the renormalisation scale; the problem was noted to be particularly severe for a full calculation of the twoloop Higgs mass via the effective potential technique in the MSSM in [22]. However, it would be interesting to explore alternative solutions to this problem.

\subsection{Limitations}

As we have stated above, the presented procedure can reproduce the Higgs mass for a wide range of SUSY models with the precision most spectrum generators provide at the moment for the MSSM. That means that we can include all two-loop corrections including the strong coupling and any superpotential or soft-parameter, but neglect those coming from electroweak couplings. However, even within this approximation there is still one remaining correction missing: the two-loop corrections to the electroweak VEV $v$. As can be seen from Eq. (10), $v$ will receive corrections of $\frac{\delta M_{Z}^{2,2 L}}{2 M_{Z}^{2, \text { pole }} f\left(\left\{g_{i}\right\}\right)}$ which in simple extensions of the MSSM will be $\frac{2 \delta M_{Z}^{2,2 L}}{M_{Z}^{2, \text { pole }}\left(g_{1}^{2}+g_{2}^{2}\right)}$. At any given loop order there will be contributions to $\delta M_{Z}^{2}$ proportional to $g_{1}^{2}, g_{2}^{2}$ (i.e. loops which do not contain any further electroweak couplings) and so there will be nonvanishing contributions to $v$ even in the limit that the electroweak gauge couplings are set to zero. These corrections then feed into the tree-level Higgs mass; in the MSSM this is not an issue because, in the gaugeless limit, they are multiplied by zero. However, in general extensions (such as the NMSSM) the tree-level Higgs mass matrix will contain non-vanishing elements proportional to $v$, and therefore there will be a corresponding two-loop correction to the Higgs mass. Since the general expression for these two loop corrections is not available in the literature, we leave the calculation and implementation of these to future work.

A further limitation is that only corrections to $\mathrm{CP}$ even states are calculated. Thus, two-loop corrections for pseudoscalars or charged Higgs bosons are not calculated by this setup at the moment.

\section{Validation}

We have cross-checked the Higgs mass using our new routines against the well-established routines of Refs. [20,21, $23,24,26]$. For this purpose, we made a few modifications to ensure that both routines run with equivalent conditions:

1. By default the new routine includes any correction from superpotential parameters and soft-terms while the routines of Refs. $[20,21,23,24,26]$ are restricted to third generation couplings in the context of the MSSM. Therefore, we set all couplings of the first and second generations of (s)quarks to zero.

2. As discussed above, we have implemented a flag to perform calculations in the gaugeless limit in which D-term contributions to the masses are neglected. We made use of this option. However, it was also necessary to ensure that both routines use the same values for the scalar and pseudo-scalar masses: even if $D$ terms in the mass matrices are neglected, the routines of Ref. [20,21,23,24,26] are usually called with values for $\mu$ and $M_{A}$ which correspond to the minimum of the potential including $D$-terms. Diagonalising the corresponding scalar and pseudo-scalar mass matrix would not give $M_{G}=M_{h}=0$. In our comparison, we therefore used the gaugeless limit and re-solved the tadpole equations in this limit. The obtained values for $\mu$ and $M_{A}$ were then used in both calculations to ensure that all masses running in the loops are identical.

The resulting Higgs mass for a variation of $m_{0}, M_{1 / 2}, \tan \beta$ and $A_{0}$ in the context of the CMSSM is shown in Fig. 2. One can see the very good agreement between the automatically generated routines by SARAH and the ones of Refs. [20,21, $23,24,26]$. There are tiny numerical differences stemming from the numerical derivation but those are negligible and have no visible impact on the Higgs mass as can bee seen from Table 1. One can also see from these numbers that there is hardly any difference in including $D$-terms to the tree-level masses used in the calculation, as expected. 

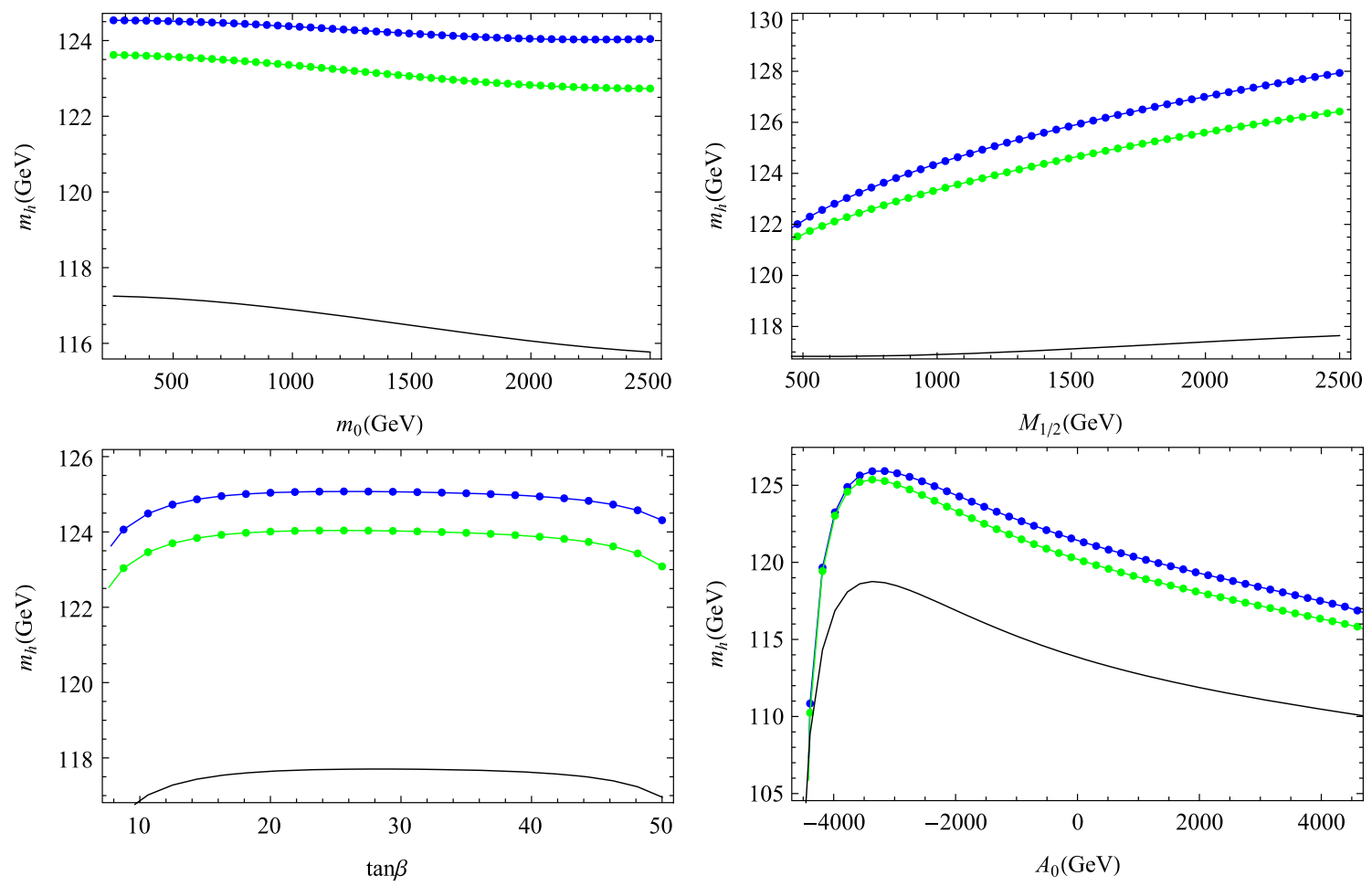

Fig. 2 The Higgs mass at one- (black) and two-loop (blue, green) in the CMSSM for a variation of $m_{0}$ (top left), $M_{1 / 2}$ (top right), $\tan \beta$ (bottom left) and $A_{0}$ (bottom right). The unvaried parameters are fixed to $m_{0}=M_{1 / 2}=1 \mathrm{TeV}, \tan \beta=10, \mu>0, A_{0}=-2 \mathrm{TeV}$. Blue shows

the Higgs mass including $\alpha_{s}\left(\alpha_{t}+\alpha_{t}\right)$ corrections while green includes all dominant two-loop corrections. The full lines are the results using the routines of Refs. $[20,21,23,24,26]$, while for the small circles the routines automatically generated by SARAH are used

Table 1 Two-loop self energies and loop-corrected masses calculated with the two numerical method to get the derivative of the effec-

$m_{0}=M_{1 / 2}=1 \mathrm{TeV}, \mu>0, \tan \beta=10, A_{0}=-2 \mathrm{TeV}$. The reference value is the one using the routines of Ref. [20,21,23,24,26] tive potential in the gaugeless limit and with full masses. We used

\begin{tabular}{|c|c|c|c|c|c|}
\hline & $\begin{array}{l}\text { Purely-numerical } \\
\text { method }\end{array}$ & $\begin{array}{l}\text { Purely-numerical } \\
\text { method (gaugeless) }\end{array}$ & $\begin{array}{l}\text { Semi-analytical } \\
\text { method }\end{array}$ & $\begin{array}{l}\text { Semi-analytical } \\
\text { method (gaugeless) }\end{array}$ & References \\
\hline$\Pi_{11}^{(2)}\left[\mathrm{GeV}^{2}\right]$ & 3475.21 & 3462.95 & 3475.18 & 3462.87 & 3460.45 \\
\hline$\Pi_{12}^{(2)}\left[\mathrm{GeV}^{2}\right]$ & -299.21 & -297.92 & -299.21 & -297.92 & -297.70 \\
\hline$\Pi_{22}^{(2)}\left[\mathrm{GeV}^{2}\right]$ & 1954.32 & 1954.06 & 1954.32 & 1954.06 & 1954.03 \\
\hline$m_{h_{1}}[\mathrm{GeV}]$ & 124.69 & 124.69 & 124.69 & 124.69 & 124.69 \\
\hline$m_{h_{2}}[\mathrm{GeV}]$ & 1963.56 & 1963.55 & 1963.56 & 1963.56 & 1963.55 \\
\hline
\end{tabular}

As a next step, we compared the two-loop results of the SARAH routines for other models with existing references: these are the $\alpha_{s}\left(\alpha_{t}+\alpha_{b}\right)$ corrections in the NMSSM [54] and in the MSSM extended by Dirac gauginos, for which analytical results and an independent code will be presented in future work. Of course, we can use also the new routines to calculate the Higgs masses in these models including the other important corrections, but the presentation and discussion of these results is beyond the scope of this paper and will be given elsewhere [99]. As example for the good agreement between our results and those of Ref. [54] we show the light Higgs mass in Fig. 3 for a variation of $\lambda$ and $\kappa$.

\section{How to use the routines}

SARAH 4.4 automatically writes the necessary Fortran routines in the SPheno output to calculate the two-loop Higgs corrections. To obtain the SPheno code for a given model download the most recent SARAH version from HepForge

http://sarah. hepforge.org/

Copy the tar-file into a directory called \$PATH in the following and extract it 


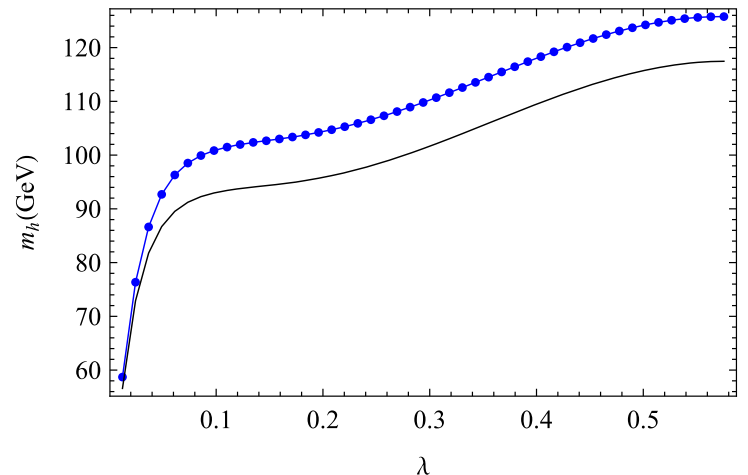

Fig. 3 The Higgs mass at one- (black) and two-loop with $\alpha_{S}\left(\alpha_{b}+\alpha_{t}\right)$ corrections (blue) in a constrained variant of the NMSSM for a variation of $\lambda$ (left) and $\kappa$ (right). The unvaried parameters are fixed to $m_{0}=$ $M_{1 / 2}=1.4 \mathrm{TeV}, \tan \beta=2.9, \mu>0, A_{0}=-1.35 \mathrm{TeV}, \lambda=0.56$,

$\operatorname{tar}-\mathrm{xf}$ SARAH-4.4.0.tar.gz

Afterwards, start Mathematica, load SARAH, run a model \$MODEL and generated the SPheno output

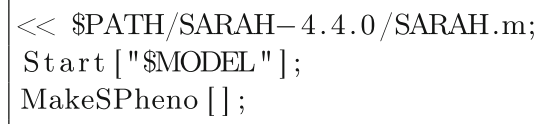

The last command initializes all necessary calculations and writes all Fortran files into the output directory of the considered model. These files can be compiled together with SPheno version 3.3.0 or later. SPheno is also available at HepForge.

http://spheno.hepforge.org/

The necessary steps to compile the new files are

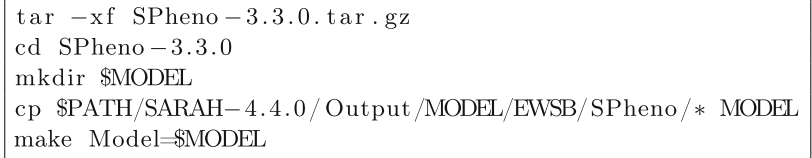

This creates a new binary bin/SPheno\$MODEL which reads all input parameters from an external file. SARAH writes a template for this input file which can be used after filling it with numbers as

. / bin /SPheno\$MODEL \$MODEL/LesHouches . in . \$MODEL

The output is written to

SPheno.spc.\$MODEL

and contains all running parameters at the renormalization scale, the loop corrected mass spectrum, two and three-body

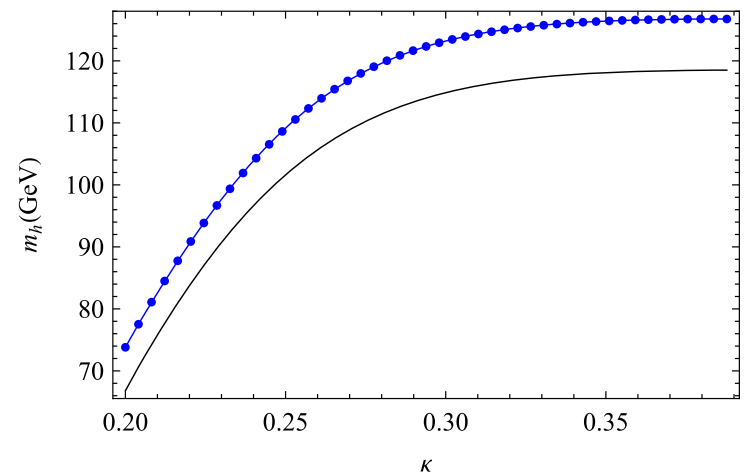

$\kappa=0.33, A_{\lambda}=-390 \mathrm{GeV}, A_{\kappa}=-280 \mathrm{GeV}, v_{S}=500 \mathrm{GeV}$. The full lines are the results using the routines of Ref. [54], while for the small circles the automatically generated routines by SARAH are used

decays as well as a prediction of precision and flavour observables. More details about the calculation of flavour observables and how to implement new observables are given in the Flavorkit manual [100]. The implementation of SARAH models in SPheno can also be automatized by using the SUSY Toolbox [101].

There are five flags which can be used in the Les Houches input file to adjust the properties of the two-loop calculation

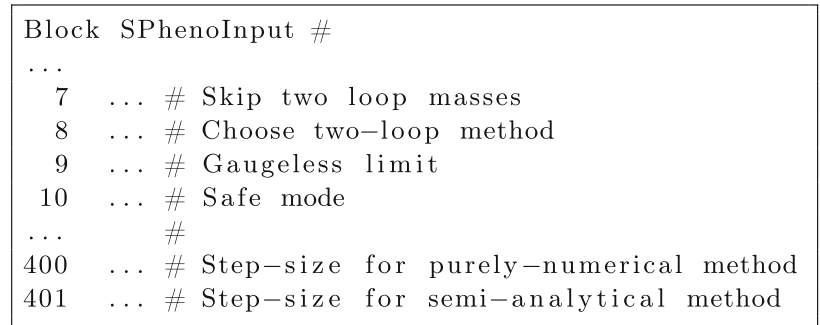

The following values are possible:

- SPhenoInput [7]:

- 0: Don't skip two-loop masses

- 1: Skip two-loop masses

- SPhenoInput [8]:

- 1: Two-loop calculation with purely numerical derivation

- 2: Two-loop calculation with analytical derivation of loop functions (default)

- 9: Use routines based on Refs. [20,21,23,24,26]

- SPhenoInput[9]:

- 0: Turn off gauge-less limit

- 1: Use gauge-less limit (default)

- SPhenoInput [10]:

- 0: Turn off the safe-mode (default) 


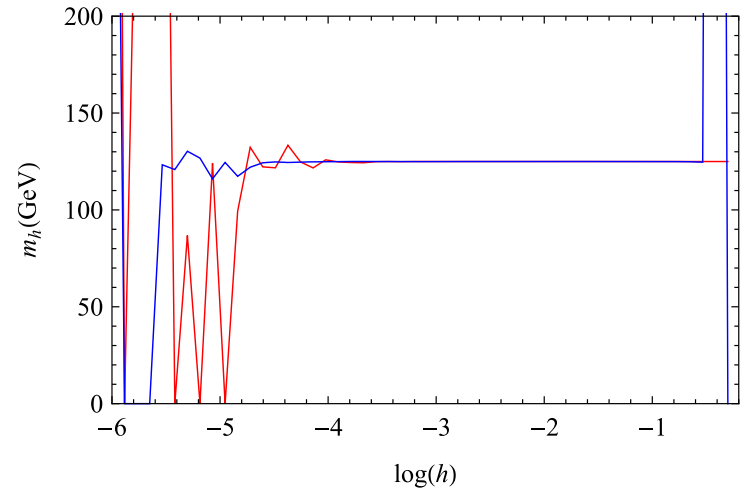

Fig. 4 Test of the numerical stability: on the left we vary the initial step-size $h$ used in the numerical derivation. The blue line corresponds to the semi-analytical approach and red for the purely numerical calculation. On the right we show the Higgs mass as function of a large

\section{- 1: Use safe-mode}

- SPhenoInput [400]: a real number (default: 0.5)

- SPhenoInput [401] : a real number (default: 0.001)

Note that the two-loop routines from Refs. [20,21,23,24,26] are not included by default in the SPheno output of SARAH. To include them, in the SPheno.m file the flag

UseHiggs2LoopMSSM = True;

has to be set.

The flags SPhenoInput [400] and SPhenoInput [401] can be used to check numerical stability of the derivation. If the step size is chosen to be too small or large the numerical derivation might suffer from some instabilites. We found that the initial step size for derivation with the fully numerical method usually needs a larger initial stepsize for the considered VEV especially for heavy SUSY spectra. The reason is that the potential is of $O\left(M_{S U S Y}^{4}\right)$ and the overall value only changes slightly when the VEVs are varied. The second method usually operates acceptably with a smaller initial step size because objects of at most order $O\left(M_{S U S Y}^{2}, M_{Z}^{2}\right)$ are derived numerically. FS: In addition, we make the approximation that in the purely numerical approach mass squareds in the loop which are smaller than $10^{-5}$ times the largest mass squared in the loop is taken to be zero. For the semi-analytical approach we take a limit of $10^{-8}$.

To give an impression of the numerical stability we show in Fig. 4 the Higgs mass for a variation of the initial step size used in two methods and for a large variation of $m_{0}$. Here, one sees that the semi-analytical method becomes stable for smaller initial step sizes as this is the case in the purely numerical calculation. Also we see that the routines are stable even for very large values of the SUSY masses.

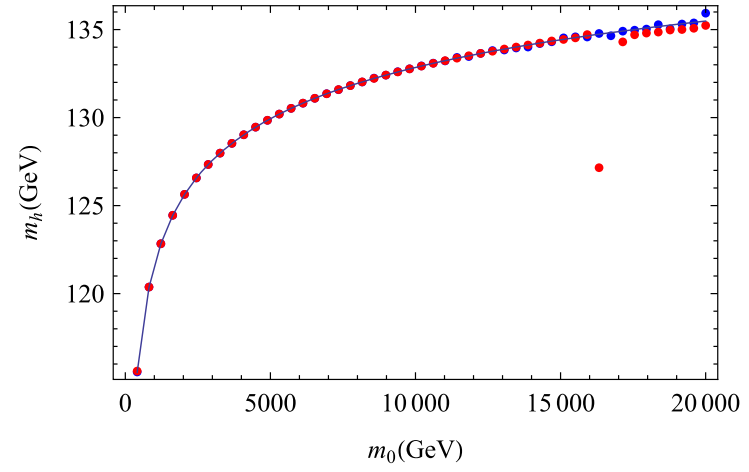

variation of $m_{0}$ (with $M_{1 / 2}=-A_{0}=m_{0}$ and $\tan \beta=10, \mu>0$ ). The solid line is based on the calculation of Refs. [20,21,23,24,26], the blue points are based on our semi-analytical method and the red ones on the purely numerical one

Here, the purely numerical method shows some instabilites for $m_{0}>15 \mathrm{TeV}$. This can be improved by changing the initial step-size to larger values. The small off-set between the purely-numerical method and the reference in the case of a very heavy SUSY spectrum is explained by our approximation to take ratios of mass squared smaller than $10^{-5}$ as zero. Because of this even the top quark is treated as massless in some loops. However, we want to stress that these instabilites appear for SUSY masses where this setup should not be used for calculations of Higgs masses. As soon as the SUSY masses are much above the EW scale the very heavy particles should be decoupled and an effective theory has to be considered [102]. Of course, this statement holds for all public versions of SUSY spectrum generators.

We also provide a "safe mode" for SPheno via flag 10: in this case SPheno starts with a large initial step size which is decreased automatically. It checks for what range of the initial step size the results are numerically stable by comparing the results obtained with different inital step sizes. If no stable range is found an error is returned. In the unlikely case that both methods suffer from numerical instabilities there is also the possibility to increase the numerical precision by passing from double to quadruple precision. For this purpose, the Makefile located in SPheno-3.3.0/srC must be changed. The line

PreDef $=$-DGENERATIONMIXING $\quad$-DONLYDOUBLE

should be replaced by

PreDef $=-$ DGENERATIONMIXING $\quad$-DQUADRUPOLE

Afterwards the entire SPheno code must be recompiled via 
make cleanall

make Model $\$$ MODEL

This will slow down the numerical evaluation significantly. However, have not found an example for resonable SUSY masses where this was necessary.

\section{Conclusion and outlook}

We have presented a fully automatised two-loop calculation of Higgs masses in supersymmetric models. The method is based on the two-loop effective potential approximation and provides the same numerical accuracy of Higgs masses in beyond MSSM models as commonly used spectrum generators do for the MSSM. That means for any BMSSM model the combination SARAH/SPheno offers now the most precise calculation for Higgs masses available. Of course, there is still much space for further improvements: we have not yet included the contributions from electroweak gauge couplings, and also masses for $\mathrm{CP}$ odd states are not yet calculated; the corrections to $v$ at two loops should be calculated and included; alternative solutions to the Goldstone boson catastrophe should be investigated. However, it is not yet clear if all of these can be accomplished in an appropriate way in the effective potential approach. Therefore, future developments will go in the direction of a diagrammatic calculation where these effects can be included in a more straightforward way-a further update of SARAH together with analytical calculations are in preparation. Nevertheless, the presented framework pushes precision studies in non-minimal SUSY models to a new level. It allows the prediction of the Higgs mass to be confronted in many SUSY models in the same way as this is done in the MSSM.

Acknowledgments We thank Stephen Martin for quickly answering our questions concerning Ref. [90]. We reserve particular thanks to Pietro Slavich for many informative discussions and pointing out the issue with the two-loop corrections to $v$.

Open Access This article is distributed under the terms of the Creative Commons Attribution License which permits any use, distribution, and reproduction in any medium, provided the original author(s) and the source are credited.

Funded by $\mathrm{SCOAP}^{3}$ / License Version CC BY 4.0

\section{Appendix A: The Two-loop effective potential in four-component notation}

The basis for our implementation is the two-loop effective potential for a general renormalizable theory, given by S. Martin [90]. His convention is the most elegant and simplest when dealing with a general theory, using only Weyl fermions $\left(\psi_{I}\right)$, real scalars $\left(R_{K}\right)$ and real vectors $\left(A_{\mu}^{a}\right)$. We will refer to it as the $\mathrm{R}$-convention ( $\mathrm{R}$ for real). However, in a specific model it is more useful to organise particles into groups, including explicitly real and complex scalars, Majorana and Dirac fermions. This is the case in the framework of SARAH/SPheno: Fermions are described by bispinors $\Psi_{i}$, which can be Dirac or Majorana, and bosons can be real or complex. We will call this the $\mathrm{C}$-convention ( $\mathrm{C}$ for complex). In this section we will slightly recast the formulae of the reference to a form suitable for implementation.

The general structure of the effective potential at two-loop can be decomposed into ten terms,

$$
\begin{aligned}
V^{(2)}= & V_{S S S}^{(2)}+V_{S S}^{(2)}+V_{F F S}^{(2)}+V_{\overline{F F} S}^{(2)}+V_{S S V}^{(2)}+V_{V S}^{(2)} \\
& +V_{V V S}^{(2)}+V_{F F V}^{(2)}+V_{\overline{F F} V}^{(2)}+V_{\text {gauge }}^{(2)} .
\end{aligned}
$$

Every contribution is described by a simple expression,

$V^{(2)} \sim$ (coupling $)^{2} \times f_{\text {loop }}\left(m_{1}^{2}, m_{2}^{2}, m_{3}^{2}\right)$, sunrise topology

$V^{(2)} \sim$ (coupling) $\times f_{\text {loop }}\left(m_{1}^{2}, m_{2}^{2}\right)$, snowman topology

in which only the prefactors must be figured out carefully. Usually, the loop functions will be abbreviated to $f_{\text {loop }}\left(m_{i}^{2}, m_{j}^{2}, m_{k}^{2}\right)=f_{\text {loop }}(i, j, k)$.

In the SARAH framework, a vertex factor for three scalars $\phi_{1}, \phi_{2}, \phi_{3}$ is understood as

vertex $=i \frac{\delta \mathcal{L}}{\delta \phi_{1} \delta \phi_{2} \delta \phi_{3}}=: i c$,

which is what is used in the textbook approach to writing down Feynman amplitudes. $\phi_{i}$ are either Weyl fermions or bosons. The vertex factor can be decomposed into a kinematic part (Lorentz indices) and a coefficient. For example, for two Dirac fermions and a vector boson, the vertex factor can be expressed as

vertex $=i\left(c_{L} P_{L}+c_{R} P_{R}\right) \gamma^{\mu}$

Here, $P_{L}$ and $P_{R}$ are the polarization operators $P_{L, R}=$ $\frac{1}{2}\left(1 \mp \gamma_{5}\right)$. The coefficients for particles $\mathrm{p} 1, \mathrm{p} 2, \mathrm{p} 3,(\mathrm{p} 4)$ can be obtained in SARAH by the command

Vertex $[\{\mathrm{p} 1, \mathrm{p} 2, \mathrm{p} 3\}]$

Vertex $[\{\mathrm{p} 1, \mathrm{p} 2, \mathrm{p} 3, \mathrm{p} 4\}]$

e.g. Vertex $[\{\mathrm{Fu}, \operatorname{bar}[\mathrm{Fu}], \mathrm{VG}\}]$ returns the vertex of $t, \bar{t}, g$.

SARAH calculates internally with 2-component spinors, but the final set of particles as used in SPheno is given in terms of Dirac spinors. The Vertex-command accepts both. If $\xi$ and $\chi$ are 2-component spinors with the same quantum numbers, a Dirac spinor can be constructed (Chiral represen- 
tation)

$\Psi=\left(\begin{array}{c}\xi \\ \chi^{\dagger}\end{array}\right), \bar{\Psi}=\Psi^{\dagger} \gamma^{0}=\left(\chi, \xi^{\dagger}\right)$

with the translation table

$\bar{\Psi}_{i} \gamma^{\mu} P_{L} \Psi_{j}=\xi_{i}^{\dagger} \bar{\sigma}^{\mu} \xi_{j}, \quad \bar{\Psi}_{i} \gamma^{\mu} P_{R} \Psi_{j}=\chi_{i} \sigma^{\mu} \chi_{j}^{\dagger}$

$\bar{\Psi}_{i} P_{L} \Psi_{j}=\chi_{i} \xi_{j}, \quad \bar{\Psi}_{i} P_{R} \Psi_{j}=\xi_{i}^{\dagger} \chi_{j}^{\dagger}$

with $\sigma^{\mu}=\left(1, \sigma^{i}\right)$ and $\bar{\sigma}^{\mu}=\left(1,-\sigma^{i}\right)$ and the Pauli matrices $\sigma^{i}$.

For each of the terms in Eq. (A.1) we will define a piece of Lagrangian such that $i c$ or $i c_{L / R}$ will match the output of a SARAH Vertex command (cf. Eq. A.4). This Lagrangian is then transformed such that the relations between these couplings and the S. Martin couplings are obvious. When using SARAH conventions, we will write lower-case indices $i, j, k$ to denote generation or color indices. When using capital letters $I, J, K$, they refer to the notation where everything is broken down to 2-component spinors $\psi_{I}=\left(\xi_{1}, \chi_{1}, \xi_{2}, \chi_{2}, \ldots\right)$ and real scalars $R_{K}=$ $\left(\varphi_{1}, \sigma_{1}, \varphi_{2}, \sigma_{2}, \ldots\right)$.

\section{A.1: $F F V$ and $\overline{F F} V$}

Given a set of fermions $\Psi_{i}$ and a vector $A_{\mu}^{a}$, the Lagrangian term is

$$
\begin{aligned}
\mathcal{L}_{F F V} & =\bar{\Psi}_{i} \gamma^{\mu}\left(c_{L} P_{L}+c_{R} P_{R}\right) \Psi_{j} A_{\mu}^{a} \\
& =c_{L} \xi_{i}^{\dagger} \bar{\sigma}^{\mu} \xi_{j} A_{\mu}^{a}+c_{R} \chi_{i} \sigma^{\mu} \chi_{j}^{\dagger} A_{\mu}^{a} \\
& =c_{L} \xi_{i}^{\dagger} \bar{\sigma}^{\mu} \xi_{j} A_{\mu}^{a}-c_{R} \chi_{j}^{\dagger} \bar{\sigma}^{\mu} \chi_{i} A_{\mu}^{a}
\end{aligned}
$$

There is no implicit sum over $i, j, k$ here. The coefficients are $c_{L / R}=c_{L / R}(i, j, a)$. The minus sign comes from the rearrangement $\chi \sigma^{\mu} \xi^{\dagger}=-\xi^{\dagger} \bar{\sigma}^{\mu} \chi$ (in signatures with mostly plus as well as mostly minus). We can arrange all 2-component spinors in a list: $\left(\psi_{I}\right)=\left(\xi_{1}, \chi_{1}, \xi_{2}, \chi_{2}, \ldots\right)$. The interaction in $\mathrm{S}$. Martin convention is given by

$$
\mathcal{L}_{F F V}=\sum_{I, J, a} g_{I J}^{a} \psi_{I}^{\dagger} \bar{\sigma}^{\mu} \psi_{J} A_{\mu}^{a} .
$$

Since this term has to be real, $g_{I J}^{a}=\left(g_{J I}^{a}\right)^{\star}$ holds. Vectors only couple left to left and right to right, so a rewriting of Eq. (A.11) is useful:

$\mathcal{L}=g_{L i j}^{a} \xi_{i}^{\dagger} \bar{\sigma}^{\mu} \xi_{j} A_{\mu}^{a}+g_{R i j}^{a} \chi_{i}^{\dagger} \bar{\sigma}^{\mu} \chi_{j} A_{\mu}^{a}$.

The connection is $g_{L i j}^{a}=g_{I=2 i-1, J=2 j-1}^{a}, g_{R i j}^{a}=g_{I=2 i, J=2 j}^{a}$. This allows to match the coefficients,

$$
\begin{aligned}
& g_{L i j}^{a}=c_{L}(i, j) \\
& g_{R i j}^{a}=-c_{R}(j, i)=-c_{R}(i, j)^{*}=-c_{R}^{*} .
\end{aligned}
$$

The original expression for $\overline{F F} V$ is given by

$V_{\overline{F F} V}^{(2)}=\frac{1}{2} g_{I J}^{a} g_{I^{\prime} J^{\prime}}^{a} M_{I I^{\prime}} M_{J J^{\prime}}^{\star} F_{\overline{F F} V}(I, J, a)$

$M_{I J}$ denotes a mass insertion, which in case of a Dirac fermion is a $2 \times 2$ block matrix, $\left(\begin{array}{cc}0 & m_{D} \\ m_{D} & 0\end{array}\right)$, or more formally expressed as $M_{I J}=m_{D i}\left(\delta_{I, 2 i-1} \delta_{J, 2 i}+\delta_{I, 2 i} \delta_{J, 2 i-1}\right)$. This allows to partially simplify:

$$
g_{I J}^{a} M_{I I^{\prime}}=\left(g_{L i j}^{a} \delta_{J, 2 j-1} \delta_{I^{\prime}, 2 i}+g_{R i j}^{a} \delta_{I^{\prime}, 2 i-1} \delta_{J, 2 j}\right) m_{i},
$$

$g_{I \prime J,}^{a} M_{J J^{\prime}}=\left(g_{L i j}^{a} \delta_{I \prime, 2 i-1} \delta_{J, 2 j}+g_{R i j}^{a} \delta_{J, 2 j-1} \delta_{I^{\prime}, 2 i}\right) m_{j}$.

The whole expression becomes

$$
\begin{aligned}
V_{\overline{F F} V}^{(2)} & =\frac{1}{2} \sum_{I, J, I^{\prime}, J^{\prime}} g_{I J}^{a} g_{I^{\prime} J^{\prime}}^{a} M_{I I^{\prime}} M_{J J^{\prime}}^{\star} F_{\overline{F F} V}\left(m_{I}^{2}, m_{J}^{2}, m_{a}^{2}\right) \\
& =\sum_{i, j} g_{L i j}^{a} g_{R i j}^{a} m_{D i} m_{D j} F_{\overline{F F} V}\left(m_{D i}^{2}, m_{D j}^{2}, m_{a}^{2}\right)
\end{aligned}
$$

Observe that for a Dirac fermion, $m_{2 i-1}=m_{2 i}=m_{D i}$. If we fix two different particles $i$ and $j$, this sum has two terms that involve these particles.

$$
\begin{aligned}
V_{\overline{F F} V, i \neq j}^{(2)} & =\left(g_{L i j}^{a} g_{R i j}^{a}+g_{L j i}^{a} g_{R j i}^{a}\right) m_{D i} m_{D j} F_{\overline{F F} V}(i, j, a) \\
& =2 \Re\left(g_{L i j}^{a} g_{R i j}^{a}\right) m_{D i} m_{D j} F_{\overline{F F} V}(i, j, a) \\
& =-2 \Re\left(c_{L} c_{R}^{*}\right) m_{D i} m_{D j} F_{\overline{F F} V}(i, j, a)
\end{aligned}
$$

If we fix the same particle $i=j$, the factor 2 disappears.

$V_{\overline{F F} V, i=j}^{(2)}=-\Re\left(c_{L} c_{R}^{*}\right) m_{D i}^{2} F_{\overline{F F} V}(i, i, a)$

Now consider Majorana particles, which are written as

$\Psi_{M i}=\left(\begin{array}{c}\xi_{i} \\ \xi_{i}^{\dagger}\end{array}\right)$

For those particles, the mass insertion matrix $M_{I J}$ has diagonal elements $m_{M i}$. Lets consider $i$ labelling a Dirac fermion and $j$ a Majorana fermion. Each mass insertion in Eq. (A.16) can be from each of these fermions. If we take only $M_{I I^{\prime}}$ to be the Dirac mass insertions and $M_{J J^{\prime}}$ the Majorana mass, this gives

$g_{L i j}^{a} g_{R i j}^{a} m_{D i} m_{M j} F_{\overline{F F} V}(i, j, a)$.

It can also be the other way round, which leads to

$g_{L j i}^{a} g_{R j i}^{a} m_{D i} m_{M j} F_{\overline{F F} V}(i, j, a)$. 
Table 2 Summary of $F F V, \overline{F F} V, F F S, \overline{F F} S$ contributions. The contribution is given by $V=k \cdot \operatorname{coup} \cdot f(i, j, k$ ) (for $\overline{F F} V, \overline{F F} S$ times $m_{i} m_{j}$ ). The table shows the product $k$. coup for various cases.
$D_{i} D_{j} c(r)$ stands for Dirac fermions with complex (real) scalars or vectors, $M_{i} M_{j} c(r)$ for Majorana fermions

\begin{tabular}{llllll}
\hline & $D_{i} D_{j} c$ & $D_{i} D_{j} r$ & $D_{i} M_{j}$ & $M_{i} M_{j} c$ & $M_{i} M_{j} r$ \\
\hline$V_{F F V}^{(2)}, i \neq j$ & $\left(\left|c_{L}\right|^{2}+\left|c_{R}\right|^{2}\right)$ & $\left(\left|c_{L}\right|^{2}+\left|c_{R}\right|^{2}\right)$ & $\left(\left|c_{L}\right|^{2}+\left|c_{R}\right|^{2}\right)$ & $|c|^{2}$ & $|c|^{2}$ \\
$V_{F F V}^{(2)}, i=j$ & $\frac{1}{2}\left(\left|c_{L}\right|^{2}+\left|c_{R}\right|^{2}\right)$ & $\frac{1}{2}\left(\left|c_{L}\right|^{2}+\left|c_{R}\right|^{2}\right)$ & - & $\frac{1}{2}|c|^{2}$ & $\frac{1}{2}|c|^{2}$ \\
$V \frac{(2)}{F F V}, i \neq j$ & $-2 \Re\left(c_{L} c_{R}^{*}\right)$ & $-2 \Re\left(c_{L} c_{R}^{*}\right)$ & $-2 \Re\left(c_{L} c_{R}^{*}\right)$ & $\Re\left(c^{2}\right)$ & $\Re\left(c^{2}\right)$ \\
$V \overline{F F V}, i=j$ & $-\Re\left(c_{L} c_{R}^{*}\right)$ & $-\Re\left(c_{L} c_{R}^{*}\right)$ & - & - & $\frac{1}{2} \Re\left(c^{2}\right)$ \\
$V_{F F S}^{(2)}, i \neq j$ & $\left(\left|c_{L}\right|^{2}+\left|c_{R}\right|^{2}\right)$ & $\left(\left|c_{L}\right|^{2}+\left|c_{R}\right|^{2}\right)$ & $\left(\left|c_{L}\right|^{2}+\left|c_{R}\right|^{2}\right)$ & $\left|c_{L}\right|^{2}+\left|c_{R}\right|^{2}$ & $|c|^{2}$ \\
$V_{F F S}^{(2)}, i=j$ & $\left(\left|c_{L}\right|^{2}+\left|c_{R}\right|^{2}\right)$ & $\frac{1}{2}\left(\left|c_{L}\right|^{2}+\left|c_{R}\right|^{2}\right)$ & - & $\frac{1}{2}\left(\left|c_{L}\right|^{2}+\left|c_{R}\right|^{2}\right)$ & $\frac{1}{2}|c|^{2}$ \\
$V_{F F S}^{(2)}, i \neq j$ & $2 \Re\left(c_{L} c_{R}^{*}\right)$ & $2 \Re\left(c_{L} c_{R}^{*}\right)$ & $2 \Re\left(c_{L} c_{R}^{*}\right)$ & $2 \Re\left(c_{L} c_{R}^{*}\right)$ & $\Re\left((c)^{2}\right)$ \\
$V \frac{(2)}{F F S}, i=j$ & $2 \Re\left(c_{L} c_{R}^{*}\right)$ & $\Re\left(c_{L} c_{R}^{*}\right)$ & - & $\Re\left(c_{L} c_{R}^{*}\right)$ & $\frac{1}{2} \Re\left(c^{2}\right)$ \\
\hline
\end{tabular}

In total we have

$$
\begin{aligned}
V_{\overline{F F} V}^{(2)} & =2 \Re\left(g_{L i j}^{a} g_{R i j}^{a}\right) m_{D i} m_{M j} F_{\overline{F F} V}(i, j, a) \\
& =-2 \Re\left(c_{L} c_{R}^{*}\right) m_{D i} m_{M j} F_{\overline{F F} V}(i, j, a) .
\end{aligned}
$$

When two Majorana fermions interact via gauge coupling, we find $c_{R}=-c_{L}^{*}$, so there is essentially just one coupling $c_{L}(i, j)$. The indices of the mass insertion $M_{I I^{\prime}}$ can again give the mass $m_{M i}$ or $m_{M j}$, so Eq. (A.16) simplifies to

$V_{\overline{F F} V}^{(2)}=\Re\left(\left(g_{L i j}^{a}\right)^{2}\right) m_{M i} m_{M j} F_{\overline{F F} V}(i, j, a)$.

In the case of equal Majorana fermions, $i=j$, we get

$V_{\overline{F F} V, i=j}^{(2)}=\frac{1}{2} \Re\left(\left(g_{L i i}^{a}\right)^{2}\right) m_{M i}^{2} F_{\overline{F F} V}(i, i, a)$.

Example: In the case of the gluinos, the gauge interaction term is usually introduced with an $i$,

$\mathcal{L}_{\mathrm{Glu}}=i g f^{a b c} \lambda^{a \dagger} \bar{\sigma}^{\mu} A_{\mu}^{b} \lambda^{c}$,

so here $g_{L, a c}^{b}=i g f^{a b c}$, which results in an overall Minus sign in the contribution. $f^{a b c}$ are the structure constants of $S U$ (3). Evaluating Eq. (A.31) for this case gives

$$
\begin{aligned}
V_{\widetilde{g}}^{(2)} & =-\frac{1}{2} g^{2} \underbrace{\left(\sum_{a, b, c=1}^{8}\left(f^{a b c}\right)^{2}\right)}_{=24}\left|M_{3}\right|^{2} F_{\overline{F F} V}\left(M_{3}^{2}, M_{3}^{2}, 0\right) \\
& =-12 g^{2}\left|M_{3}^{2}\right| F_{\overline{F F} V}\left(M_{3}^{2}, M_{3}^{2}, 0\right)
\end{aligned}
$$

This shows the emergence of the color factor of 24 . The result matches that of [25, Eq. (3.74)]. Now consider the $F F V$ contributions, given by

$$
V_{F F V}^{(2)}=\frac{1}{2} \sum_{I, J, a}\left|g_{I J}^{a}\right|^{2} F_{F F V}(I, J, a) \text {. }
$$

If one of the fermions is Dirac and the other Majorana (MD) (or both Dirac, DD), there are two couplings $g_{L}, g_{R}$ involved. For a fixed pair $I \neq J$, there are two equal terms in the sum in Eq. (A.35).

$V_{F F V, i \neq j}^{(2)}=\left(\left|g_{L i j}^{a}\right|^{2}+\left|g_{R i j}^{a}\right|^{2}\right) F_{F F V}(i, j, a)$ DD or MD

$V_{F F V, i \neq j}^{(2)}=\left|g_{L i j}^{a}\right|^{2} F_{F F V}(i, j, a) \quad \mathrm{MM}$

If the fermions are equal, the sum only collects terms $I=J$ and there is a factor of 2 less.

$$
\begin{aligned}
& V_{F F V, i=j}^{(2)}=\frac{1}{2}\left(\left|g_{L i i}^{a}\right|^{2}+\left|g_{R i i}^{a}\right|^{2}\right) F_{F F V}(i, i, a) \\
& V_{F F V, i=j}^{(2)}=\frac{1}{2}\left|g_{L i i}^{a}\right|^{2} F_{F F V}(i, i, a) \mathrm{MM}
\end{aligned}
$$

All the different expressions are summarized in Table 2 together with $F F S / \overline{F F} S$ discussed next.

\section{A.2: $F F S$ and $\overline{F F} S$}

These contributions are similar in structure to $F F V, \overline{F F} V$. Consider a set of 4-component fermions $\Psi_{i}$ and scalars $\phi_{k}=$ $\left(\varphi_{k}+i \sigma_{k}\right) / \sqrt{2}$ and constants $c_{L / R}=c_{L / R}(i, j, k)$. Again, for simplicity, consider $i, j, k$ fixed.

$$
\begin{aligned}
\mathcal{L}_{F F S}= & -\bar{\Psi}_{i}\left(c_{L} P_{L}+c_{R} P_{R}\right) \Psi_{j} \cdot \phi_{k}+\text { h.c. } \\
= & -\left(c_{L} \chi_{i} \xi_{j}+c_{R} \xi_{i}^{\dagger} \chi_{j}^{\dagger}\right) \phi_{k}+\text { h.c. } \\
= & -\left(c_{L} \chi_{i} \xi_{j} \phi_{k}+c_{R}^{*} \xi_{i} \chi_{j} \phi_{k}^{*}\right)+\text { h.c. } \\
= & -\left(\frac{c_{L}}{\sqrt{2}} \chi_{i} \xi_{j} R_{k}+\frac{i c_{L}}{\sqrt{2}} \chi_{i} \xi_{j} \sigma_{k}\right. \\
& \left.+\frac{c_{R}^{*}}{\sqrt{2}} \chi_{j} \xi_{i} R_{k}+\frac{-i c_{R}^{*}}{\sqrt{2}} \chi_{j} \xi_{i} \sigma_{k}\right)+ \text { h.c. }
\end{aligned}
$$

Note that scalars couple left to right handed parts. In Rconvention all scalars are real, labelled as $R_{K}=\left(\varphi_{1}, \sigma_{1}\right.$, 
$\left.\varphi_{2}, \sigma_{2} \ldots\right)$. In this convention, the interaction is given by

$$
\begin{aligned}
\mathcal{L}_{F F S}= & -\frac{1}{2} \sum_{I, J, K} y^{I J K} \psi_{I} \psi_{J} R_{K}+\text { h.c. } \\
= & -\frac{1}{2} \sum_{I, K} y^{I I K}\left(\psi_{I}\right)^{2} R_{K} \\
& -\sum_{I<J, K} y^{I J K} \psi_{I} \psi_{J} R_{K}+\text { h.c. }
\end{aligned}
$$

so the coefficient of every term in Eq. (A.43) corresponds to a different $y^{I J K}$ with $I<J$. The two-loop contributions to $F F S$ and $\overline{F F} S$ are

$$
\begin{aligned}
& V_{F F S}^{(2)}=\frac{1}{2} \sum_{I, J, K}\left|y^{I J K}\right|^{2} F_{F F S}(I, J, K) \\
& V_{\overline{F F} S}^{(2)}=\frac{1}{4} \sum_{I, J, K} y^{I J K} y^{I^{\prime} J^{\prime} k} M_{I I^{\prime}}^{*} M_{J J^{\prime}}^{*} F_{\overline{F F} S}(I, J, K)+\text { h.c. }
\end{aligned}
$$

The sum runs freely over $I, J, K$. When evaluating this sum, a symmetry factor of 2 appears in Eq. (A.46) because for each pair $I \neq J$ there is an equal term with $I, J$ interchanged. In the $\overline{F F} S$ case, $I$ can take 4 different indices, each of which give the same expression in the sum.

$$
\begin{aligned}
V_{F F S}^{(2)}= & \left(\left|\frac{c_{L}}{\sqrt{2}}\right|^{2}+\left|\frac{i c_{L}}{\sqrt{2}}\right|^{2}+\left|\frac{c_{R}^{*}}{\sqrt{2}}\right|^{2}\right. \\
& \left.+\left|\frac{-i c_{R}^{*}}{\sqrt{2}}\right|^{2}\right) F_{F F S}(i, j, k) \\
= & \left(\left|c_{L}\right|^{2}+\left|c_{R}\right|^{2}\right) F_{F F S}(i, j, k) \\
V_{\overline{F F} S}^{(2)}= & \left(\frac{c_{L}}{\sqrt{2}} \frac{c_{R}^{*}}{\sqrt{2}}+\frac{i c_{L}}{\sqrt{2}} \frac{-i c_{R}^{*}}{\sqrt{2}}\right) \\
& \times m_{D i} m_{D j} F_{\overline{F F} S}(i, j, k)+\mathrm{hc} \\
= & 2 \Re\left(c_{L} c_{R}^{*}\right) m_{D i} m_{D j} F_{\overline{F F} S}(i, j, k)
\end{aligned}
$$

If the scalar is real instead of complex, the $\sqrt{2}$ will disappear everywhere and $\sigma_{k}$ can be dropped. This leads to the exact same results as Eqs. (A.49) and (A.51). If there is one Dirac and one Majorana fermion, we can set $\chi_{j}=\xi_{j}$ in Eq. (A.43). The result also stays the same, Eqs. (A.49) and (A.51). Considering two Majorana fermions, i.e. setting $\chi_{j}=\xi_{j}$ and $\chi_{i}=\xi_{i}$, we get

$$
\mathcal{L}_{F F S}=-\left(\frac{c_{L}+c_{R}^{*}}{\sqrt{2}} \xi_{i} \xi_{j} R_{k}+i \frac{c_{L}-c_{R}^{*}}{\sqrt{2}} \xi_{i} \xi_{j} \sigma_{k}\right)+\text { h.c.. }
$$

Evaluating the contribution to the potential gives

$$
\begin{aligned}
V_{F F S}^{(2)}= & \left(\left|\frac{c_{L}+c_{R}^{*}}{\sqrt{2}}\right|^{2}+\left|i \frac{c_{L}-c_{R}^{*}}{\sqrt{2}}\right|^{2}\right) F_{F F S}(i, j, k) \\
= & \left(\left|c_{L}\right|^{2}+\left|c_{R}\right|^{2}\right) F_{F F S}(i, j, k) \\
V_{\overline{F F} S}^{(2)}= & \frac{1}{2} \sum_{I<J}\left(y^{I J K}\right)^{2} m_{M i} m_{M j} f_{\overline{F F} S}(i, j, k)+\text { h.c. } \\
= & \frac{1}{2}\left(\left(\frac{c_{L}+c_{R}^{*}}{\sqrt{2}}\right)^{2}+\left(i \frac{c_{L}-c_{R}^{*}}{\sqrt{2}}\right)^{2}\right) \\
& \times m_{M i} m_{M j} f_{\overline{F F} S}(i, j, k)+\text { h.c. } \\
= & 2 \Re\left(c_{L} c_{R}^{*}\right) m_{M i} m_{M j} f_{\overline{F F} S}(i, j, k)
\end{aligned}
$$

If there are two Majoranas and one real scalar, the interaction would be

$$
\begin{aligned}
\mathcal{L} & =-\bar{\Psi}_{i}\left(c_{L} P_{L}+c_{R} P_{R}\right) \Psi_{j} R_{k} \\
& =-c_{L} \xi_{i} \xi_{j} R_{k}+c_{R} \xi_{i}^{\dagger} \xi_{j}^{\dagger} R_{k} .
\end{aligned}
$$

The complex conjugate is not needed, because the righthanded part already serves that purpose, if $c_{R}=c_{L}^{*}$ is imposed. The contribution to $V^{(2)}$ is

$$
\begin{aligned}
V_{F F S}^{(2)} & =\left|c_{L}\right|^{2} F_{F F S}(i, j, k) \\
V_{\overline{F F S}}^{(2)} & =\frac{1}{2}\left(c_{L}\right)^{2} m_{M i} m_{M j} F_{\overline{F F} S}(i, j, k)+\text { h.c. } \\
& =\Re\left(\left(c_{L}\right)^{2}\right) m_{M i} m_{M j} F_{\overline{F F} S}(i, j, k)
\end{aligned}
$$

In the case of equal Dirac fermions and a complex scalar, the interaction Lagrangian will be

$$
\begin{aligned}
\mathcal{L}_{F F S} & =-\bar{\Psi}_{i}\left(c_{L} P_{L}+c_{R} P_{R}\right) \Psi_{i} \cdot \phi_{k}+\text { h.c. } \\
& =-\left(\frac{c_{L}+c_{R}^{*}}{\sqrt{2}} \chi_{i} \xi_{i} R_{k}+\frac{i\left(c_{L}-c_{R}^{*}\right)}{\sqrt{2}} \chi_{i} \xi_{i} \sigma_{k}\right)+\text { h.c. },
\end{aligned}
$$

which results in

$$
\begin{aligned}
V_{F F S}^{(2)}= & \left(\left|c_{L}\right|^{2}+\left|c_{R}\right|\right) F_{F F S}(i, i, k) \\
V_{\overline{F F} S}^{(2)}= & \frac{1}{2}\left(\left(\frac{c_{L}+c_{R}^{*}}{\sqrt{2}}\right)^{2}+\left(\frac{i\left(c_{L}-c_{R}^{*}\right)}{\sqrt{2}}\right)^{2}\right) \\
& \times m_{D i}^{2} F_{\overline{F F} S}(i, i, k)+\text { h.c. } \\
= & 2 \Re\left(c_{L} c_{R}^{*}\right) m_{D i}^{2} F_{\overline{F F} S}(i, i, k) .
\end{aligned}
$$

In the case of equal Dirac fermions and a real scalar, the interaction Lagrangian is

$$
\begin{aligned}
\mathcal{L}_{F F S} & =-\bar{\Psi}_{i}\left(c_{L} P_{L}+c_{R} P_{R}\right) \Psi_{i} \cdot R_{k}+\text { h.c. } \\
& =-\left(c_{L} \chi_{i} \xi_{i}+c_{R} \chi_{i}^{\dagger} \xi_{i}^{\dagger}\right) R_{k}
\end{aligned}
$$


where again $c_{R}=c_{L}^{*}$ is required. This leads to

$$
\begin{aligned}
V_{F F S}^{(2)}= & \left|c_{L}\right|^{2} F_{F F S}(i, i, k)=\frac{1}{2}\left(\left|c_{L}\right|^{2}+\left|c_{R}\right|^{2}\right) \\
& \times F_{F F S}(i, i, k) \\
V_{\overline{F F S}}^{(2)}= & \frac{1}{2}\left(c_{L}\right)^{2} m_{D i}^{2} F_{\overline{F F} S}(i, i, k)+\text { h.c. } \\
= & \Re\left(\left(c_{L}\right)^{2}\right) m_{D i}^{2} F_{\overline{F F} S}(i, i, k) \\
= & \Re\left(c_{L} c_{R}^{*}\right) m_{D i}^{2} F_{\overline{F F} S}(i, i, k)
\end{aligned}
$$

Finally, there is the case of equal Majorana fermions and a complex scalar, where we have to start with a factor of $\frac{1}{2}$ in $\mathcal{L}$

$$
\begin{aligned}
\mathcal{L}_{F F S} & =-\frac{1}{2} \bar{\Psi}_{i}\left(c_{L} P_{L}+c_{R} P_{R}\right) \Psi_{i} \phi_{k}+\text { h.c. } \\
& =-\frac{1}{2}\left(c_{L} \xi_{i}^{2}+c_{R}\left(\xi_{i}^{\dagger}\right)^{2}\right) \phi_{k}+\text { h.c. } \\
& =-\frac{1}{2}\left(\frac{c_{L}+c_{R}^{*}}{\sqrt{2}} \xi_{i}^{2} R_{k}+i \frac{c_{L}-c_{R}^{*}}{\sqrt{2}} \xi_{i}^{2} \sigma_{k}\right)+\text { h.c.. }
\end{aligned}
$$

This time there is no symmetry factor in the sum over $I, J$, so we end up with

$$
\begin{aligned}
V_{F F S}^{(2)} & =\frac{1}{2}\left(\left|c_{L}\right|^{2}+\left|c_{R}\right|^{2}\right) F_{F F S}(i, j, k) \\
V_{\overline{F F S}}^{(2)} & =\frac{1}{4}\left(2 c_{L} c_{R}^{*}\right) m_{M i}^{2} F_{\overline{F F} S}(i, i, k)+\text { h.c. } \\
& =\Re\left(\left(c_{L} c_{R}^{*}\right) m_{M i}^{2} F_{\overline{F F} S}(i, i, k) .\right.
\end{aligned}
$$

If the scalar is real, we have $c_{L}=c_{R}^{*}$ and

$$
\begin{aligned}
\mathcal{L}_{F F S} & =-\frac{1}{2} \bar{\Psi}_{i}\left(c_{L} P_{L}+c_{R} P_{R}\right) \Psi_{i} R_{k} \\
& =-\frac{1}{2} c_{L} \xi_{i}^{2} R_{k}+\text { h.c. } \\
\Rightarrow V_{F F S}^{(2)} & =\frac{1}{2}\left|c_{L}\right|^{2} F_{F F S}(i, i, k) \\
\Rightarrow V_{\overline{F F} S}^{(2)} & =\frac{1}{4}\left(c_{L}^{2}\right) m_{M i}^{2} F_{\overline{F F} S}(i, i, k)+\text { h.c. } \\
& =\frac{1}{2} \Re\left(c_{L}^{2}\right) F_{\overline{F F} S}(i, i, k)
\end{aligned}
$$

\section{A.3: SSS}

In the R-convention this interaction is given by

$$
\begin{aligned}
\mathcal{L}= & -\frac{1}{6} \lambda_{i j k} R_{i} R_{j} R_{k} \\
= & \sum_{i}\left(-\frac{1}{6} \lambda_{i i i}\right) R_{i}^{3}+\sum_{i \neq j}\left(-\frac{1}{2} \lambda_{i j j}\right) R_{i} R_{j}^{2} \\
& +\sum_{i<j<k}\left(-\lambda_{i j k}\right) R_{i} R_{j} R_{k}
\end{aligned}
$$

with three real scalars and $\lambda_{i j k}$ symmetric. The contribution to $V$ can be split up in a similar way,

$$
\begin{aligned}
V_{S S S}= & \frac{1}{12} \sum_{i j k}\left(\lambda_{i j k}\right)^{2} F_{S S S}(i, j, k) \\
= & \sum_{i<j<k} \frac{1}{2}\left(\lambda_{i j k}\right)^{2} F_{S S S}(i, j, k) \\
& +\sum_{i \neq j} \frac{1}{4}\left(\lambda_{i j j}\right)^{2} F_{S S S}(i, j, j) \\
& +\sum_{i} \frac{1}{12}\left(\lambda_{i i i}\right)^{2} F_{S S S}(i, i, i)
\end{aligned}
$$

Consider complex scalars $\phi_{i}=\left(R_{i}+i I_{i}\right) / \sqrt{2}$,

$$
\begin{aligned}
\mathcal{L}= & c \phi_{1} \phi_{2} \phi_{3}+\mathrm{cc} \\
= & \frac{c}{2 \sqrt{2}}\left(R_{1} R_{2} R_{3}-\left(I_{1} I_{2} R_{3}+I_{2} I_{3} R_{1}+I_{3} I_{1} R_{2}\right)\right. \\
& \left.-i\left(I_{1} I_{2} I_{3}-\left(R_{1} R_{2} I_{3}+R_{2} R_{3} I_{1}+R_{3} R_{1} I_{2}\right)\right)\right)+\mathrm{cc}
\end{aligned}
$$

$$
=\frac{c+c^{*}}{2 \sqrt{2}}\left(R_{1} R_{2} R_{3}-\left(I_{1} I_{2} R_{3}+I_{2} I_{3} R_{1}+I_{3} I_{1} R_{2}\right)\right)
$$

$$
\begin{aligned}
& +(-i) \frac{c-c^{*}}{2 \sqrt{2}}\left(I_{1} I_{2} I_{3}-\left(R_{1} R_{2} I_{3}+R_{2} R_{3} I_{1}+R_{3} R_{1} I_{2}\right)\right) \\
= & \frac{\Re c}{\sqrt{2}}\left(R_{1} R_{2} R_{3}-\left(I_{1} I_{2} R_{3}+I_{2} I_{3} R_{1}+I_{3} I_{1} R_{2}\right)\right) \\
& +\frac{\Im c}{\sqrt{2}}\left(I_{1} I_{2} I_{3}-\left(R_{1} R_{2} I_{3}+R_{2} R_{3} I_{1}+R_{3} R_{1} I_{2}\right)\right)
\end{aligned}
$$

Identifying the particles $R_{1}, R_{2}, R_{3}, I_{1}, I_{2}, I_{3}$ with labels $1 \ldots 6$, we get

$$
\begin{aligned}
& \frac{\Re(c)}{\sqrt{2}}=-\lambda_{123}=\lambda_{453}=\lambda_{561}=\lambda_{642} \\
& \frac{\Im(c)}{\sqrt{2}}=-\lambda_{456}=\lambda_{126}=\lambda_{234}=\lambda_{315}
\end{aligned}
$$

The effective potential contribution is

$$
\begin{aligned}
V_{S S S}= & \frac{1}{12}\left(\lambda_{i j k}\right)^{2} F_{S S S}(i, j, k) \\
= & \sum_{i<j<k} \frac{1}{2}\left(\lambda_{i j k}\right)^{2} F_{S S S}(i, j, k)=\left(\Re(c)^{2}+\Im(c)^{2}\right) \\
& \times F_{S S S}\left(m_{1}^{2}, m_{2}^{2}, m_{3}^{2}\right)=|c|^{2} F_{S S S}\left(m_{1}^{2}, m_{2}^{2}, m_{3}^{2}\right)
\end{aligned}
$$


Now consider one real scalar, $\phi_{3}=R_{3} \in \mathbb{R}$.

$$
\begin{aligned}
\mathcal{L}= & c \phi_{1} \phi_{2} \phi_{3}+\mathrm{cc}=\frac{c}{2}\left(R_{1} R_{2}-I_{1} I_{2}+i\left(R_{1} I_{2}\right.\right. \\
& \left.\left.+R_{2} I_{1}\right)\right) R_{3}+\mathrm{cc} \\
= & \Re(c)\left(R_{1} R_{2}-I_{1} I_{2}\right) R_{3}-\Im(c)\left(R_{1} I_{2}+R_{2} I_{1}\right) R_{3}
\end{aligned}
$$

The remaining five real scalars $\left(R_{1}, R_{2}, R_{3}, I_{1}, I_{2}\right)$ are labelled $1 \ldots 5$.

$$
\begin{aligned}
& \Re(c)=-\lambda_{123}=\lambda_{345} \\
& \Im(c)=\lambda_{134}=\lambda_{234}
\end{aligned}
$$

Plugging this into Eq. (A.87), we get

$$
\begin{aligned}
V_{S S S} & =\frac{1}{12}\left(\lambda_{i j k}\right)^{2} F_{S S S}(i, j, k) \\
& =\sum_{i<j<k} \frac{1}{2}\left(\lambda_{i j k}\right)^{2} F_{S S S}(i, j, k) \\
& =\left(\Re(c)^{2}+\Im(c)^{2}\right) F_{S S S}\left(m_{1}^{2}, m_{2}^{2}, m_{3}^{2}\right) \\
& =|c|^{2} F_{S S S}\left(m_{1}^{2}, m_{2}^{2}, m_{3}^{2}\right)
\end{aligned}
$$

which is the same result as Eq. (A.96). There is an additional factor of 2 in the coupling, but there are only half the number of independent $\lambda$ 's. Now, in the case of two real fields $\phi_{2}, \phi_{3}$ and one complex field $\phi_{1}$,

$$
\begin{aligned}
\mathcal{L}= & c \phi_{1} \phi_{2} \phi_{3}+\mathrm{cc}=\frac{c}{\sqrt{2}}\left(R_{1}+i I_{1}\right) R_{2} R_{3}+\mathrm{cc} \\
= & \sqrt{2} \Re(c) R_{1} R_{2} R_{3}+\sqrt{2} \Im(c) I_{1} R_{2} R_{3} \\
\Rightarrow & \sqrt{2} \Re(c)=-\lambda_{123} \\
& \times \sqrt{2} \Im(c)=\lambda_{234},
\end{aligned}
$$

there is again a factor of 2 and half the number of real field combinations. The result stays as in Eq. (A.96):

$$
V_{S S S}=|c|^{2} F_{S S S}\left(m_{1}^{2}, m_{2}^{2}, m_{3}^{2}\right) \text {. }
$$

In the case of three real fields, $c$ is real from the start and $+\mathrm{cc}$ can be omitted. There is only one $\lambda_{123}=-c$,

$V_{S S S}=\frac{1}{2}(c)^{2} F_{S S S}\left(m_{1}^{2}, m_{2}^{2}, m_{3}^{2}\right)$.

Now consider two equal complex scalars, $\phi_{2}=\phi_{3}$.

$$
\begin{aligned}
\mathcal{L}= & \frac{c}{2} \phi_{1} \phi_{2}^{2}+\mathrm{cc} \\
= & \frac{\Re(c)}{2 \sqrt{2}}\left(R_{1} R_{2}^{2}-\left(2 I_{1} I_{2} R_{2}+I_{2}^{2} R_{1}\right)\right) \\
+ & \frac{\Im(c)}{2 \sqrt{2}}\left(I_{1} I_{2}^{2}-\left(2 R_{1} R_{2} I_{2}+R_{2}^{2} I_{1}\right)\right) \\
\Rightarrow & \frac{\Re(c)}{\sqrt{2}}=-\lambda_{122}=-\lambda_{155}=\lambda_{245} \\
& \quad \times \frac{\Im(c)}{\sqrt{2}}=-\lambda_{455}=\lambda_{125}=\lambda_{224}
\end{aligned}
$$

Plugging this into Eq. (A.87), we obtain

$$
\begin{aligned}
V_{S S S}= & \sum_{i \neq j} \frac{1}{4}\left(\lambda_{i j j}\right)^{2} F_{S S S}(i, j, j) \\
& +\sum_{i<j<k} \frac{1}{2}\left(\lambda_{i j k}\right)^{2} F_{S S S}(i, j, k) \\
= & \frac{1}{2}|c|^{2} F_{S S S}\left(m_{1}^{2}, m_{2}^{2}, m_{2}^{2}\right)
\end{aligned}
$$

with a factor of $\frac{1}{2}$ compared to Eq. (A.96). If $\phi_{1}=R_{1}$ is real instead of complex, $\mathcal{L}$ reads

$$
\begin{aligned}
\mathcal{L}= & \frac{c}{2} R_{1} \phi_{2}^{2}+\mathrm{cc} \\
= & \frac{\Re(c)}{2}\left(R_{1} R_{2}^{2}-R_{1} I_{2}^{2}\right)-\Im(c) R_{1} R_{2} I_{2} \\
\Rightarrow & \Re(c)=-\lambda_{133}=+\lambda_{144} \\
& \Im(c)=-\lambda_{134} \\
\Rightarrow V_{S S S}= & \sum_{i \neq j} \frac{1}{4}\left(\lambda_{i j j}\right)^{2} F_{S S S}(i, j, j) \\
& +\sum_{i<j<k} \frac{1}{2}\left(\lambda_{i j k}\right)^{2} F_{S S S}(i, j, k) \\
= & \frac{1}{2}|c|^{2} F_{S S S}\left(m_{1}^{2}, m_{2}^{2}, m_{2}^{2}\right)
\end{aligned}
$$

In the case of two equal real scalars $R_{2}=R_{3}$ and one complex scalar $\phi_{1}$, we get

$$
\begin{aligned}
\mathcal{L} & =\frac{c}{2} \phi_{1} R_{2}^{2}+\mathrm{cc} \\
& =\frac{\Re(c)}{\sqrt{2}}\left(R_{1} R_{2}^{2}\right)+\frac{\Im(c)}{\sqrt{2}}\left(I_{1} I_{2}^{2}\right) \\
& \Rightarrow \frac{\Re(c)}{\sqrt{2}}=-\frac{1}{2} \lambda_{122}, \quad \frac{\Im(c)}{\sqrt{2}}=-\frac{1}{2} \lambda_{455}
\end{aligned}
$$

and

$$
\begin{aligned}
V_{S S S} & =\sum_{i \neq j} \frac{1}{4}\left(\lambda_{i j j}\right)^{2} F_{S S S}(i, j, j) \\
& =\frac{1}{2}|c|^{2} F_{S S S}\left(m_{1}^{2}, m_{2}^{2}, m_{2}^{2}\right) .
\end{aligned}
$$

Turning $\phi_{1}$ into a real scalar will produce only one term $\left(\frac{c}{2} R_{1} R_{2}^{2}\right)$ with a real $c=-\lambda_{122}$. This results in

$$
\begin{aligned}
V_{S S S} & =\sum_{i \neq j} \frac{1}{4}\left(\lambda_{i j j}\right)^{2} F_{S S S}(i, j, j) \\
& =\frac{1}{4}|c|^{2} F_{S S S}\left(m_{1}^{2}, m_{2}^{2}, m_{2}^{2}\right)
\end{aligned}
$$


Table 3 Prefactors for $S S S$ contributions. The contribution is given by $V_{S S S}^{(2)}=k \cdot|c|^{2} F_{S S S}\left(m_{1}^{2}, m_{2}^{2}, m_{3}^{2}\right)$, where $m_{i}$ is the mass of $\phi_{i}$. The table shows $k$ for various cases

\begin{tabular}{llll}
\hline Fields & All different & Two equal & All equal $\left(\phi_{1}=\phi_{2}=\phi_{3}\right)$ \\
\hline$\phi_{1,2,3} \in \mathbb{C}$ & 1 & $1 / 2$ & $1 / 6$ \\
$\phi_{1,2} \in \mathbb{C}, \phi_{3} \in \mathbb{R}$ & 1 & $1 / 2$ & - \\
$\phi_{1} \in \mathbb{C}, \phi_{2,3} \in \mathbb{R}$ & 1 & $1 / 2$ & - \\
$\phi_{1,2,3} \in \mathbb{R}$ & $1 / 2$ & $1 / 4$ & $1 / 12$ \\
\hline
\end{tabular}

Consider three equal complex scalars $\phi_{1}=\phi_{2}=\phi_{3}$.

$\mathcal{L}=\frac{c}{6} \phi_{1}^{3}+\mathrm{cc}$

$=\frac{\Re(c)}{6 \sqrt{2}}\left(R_{1}^{3}-3 R_{1} I_{1}^{2}\right)-\frac{\Im(c)}{6 \sqrt{2}}\left(3 R_{1} I_{1}^{2}-I_{1}^{3}\right)$

$\Rightarrow \frac{\Re(c)}{\sqrt{2}}=-\lambda_{111}=\lambda_{122}$

$\frac{\Im(c)}{\sqrt{2}}=-\lambda_{222}=\lambda_{112}$

$$
\begin{aligned}
\Rightarrow & V_{S S S}=\left(\frac{1}{8}+\frac{1}{24}\right)|c|^{2} F_{S S S}\left(m^{2}, m^{2}, m^{2}\right) \\
& =\frac{1}{6}|c|^{2} F_{S S S}\left(m^{2}, m^{2}, m^{2}\right)
\end{aligned}
$$

At last, if there are three equal real scalars, we get

$$
\begin{aligned}
\mathcal{L} & =\frac{c}{6} R_{1}^{3} \\
& \Rightarrow c=-\lambda_{111} \\
& \Rightarrow V_{S S S}=\sum_{i} \frac{1}{12}\left(\lambda_{i i i}\right)^{2} F_{S S S}\left(m^{2}, m^{2}, m^{2}\right) .
\end{aligned}
$$

All these results are summarized in Table 3.

\section{A.4: SS}

The $S S$ contribution is given by

$V_{S S}^{(2)}=\frac{1}{8} \sum_{i j} \lambda^{i i j j} F_{S S}\left(m_{i}^{2}, m_{j}^{2}\right)$

In the R-convention this interaction is described by

$\mathcal{L}=-\frac{1}{24} \sum_{i j k l} \lambda^{i j k l} R_{i} R_{j} R_{K} R_{l}$

with a real and completely symmetric $\lambda^{i j k l}$. Picking out only terms where $i=j$ and $k=l$ (both fixed), the sum reads

$\mathcal{L}=-\frac{1}{4} \lambda^{i i j j} R_{i}^{2} R_{j}^{2} \quad($ nosum,$i \neq j)$

If all four scalars are equal, the term is just

$\mathcal{L}=-\frac{1}{24} \lambda^{i i i i} R_{i}^{4} \quad$ (no sum)
Because there are only two scalars in total in the loop, we only have to distinguish the cases of different scalars and equal scalars. In the $\mathrm{C}$-convention with two charged scalars $\phi_{1}, \phi_{2}$ we have

$\mathcal{L}=c\left|\phi_{1}\right|^{2}\left|\phi_{2}\right|^{2}$

$\mathcal{L}=\frac{c}{4}\left|\phi_{1}\right|^{4} \quad$ equal scalars

where $c$ is the vertex factor in both cases. Introducing $\phi_{i}=$ $\left(R_{i}+i \sigma_{i}\right) / \sqrt{2}$, this leads to

$\mathcal{L}=\frac{c}{4}\left(R_{1}^{2}+\sigma_{1}^{2}\right)\left(R_{2}^{2}+\sigma_{2}^{2}\right)$

$\mathcal{L}=\frac{c}{16}\left(R_{1}^{4}+\sigma_{1}^{4}+2 R_{1}^{2} \sigma_{1}^{2}\right) \quad$ equal scalars

With this equation, the conventions can be matched. All real scalars $\left(R_{1}, R_{2}, \sigma_{1}, \sigma_{2}\right)$ can be labelled with indices $1,2,3,4$. The coefficients are

$-c=\lambda^{1122}=\lambda^{1144}=\lambda^{3322}=\lambda^{3344}$ different scalars

$-c=\frac{2}{3} \lambda^{1111}=\frac{2}{3} \lambda^{2222}=2 \lambda^{1122}$ equal scalars

Now simplify the potential contribution Eq. (A.137) for complex different scalars. There is a factor of 2 because of symmetry in $i, j$.

$$
\begin{aligned}
V_{S S}^{(2)} & =\frac{1}{8} \lambda^{i i j j} F_{S S}(i, j) \\
& =2 \cdot \frac{1}{8}\left(\lambda^{1122}+\lambda^{1144}+\lambda^{3322}+\lambda^{3344}\right) F_{S S}(i, j) \\
& =-c F_{S S}(i, j)
\end{aligned}
$$

Now repeat the calculation for two equal complex scalars:

$$
\begin{aligned}
V_{S S}^{(2)} & =\frac{1}{8} \lambda^{i i j j} F_{S S}(i, j) \\
& =\frac{1}{8}\left(\lambda^{R R R R}+\lambda^{\sigma \sigma \sigma \sigma}+2 \lambda^{R R \sigma \sigma}\right) F_{S S}(i, i) \\
& =-\frac{1}{8}\left(\frac{3}{2} c+\frac{3}{2} c+c\right) F_{S S}(i, i) \\
& =-\frac{1}{2} c F_{S S}(i, i)
\end{aligned}
$$

For one real $\left(R_{2}\right)$ and one complex scalar, we get

$$
\begin{aligned}
\mathcal{L} & =\frac{c}{2}\left|\phi_{1}\right|^{2} R_{2}^{2} \\
& =\frac{c}{4}\left(R_{1}^{2}+\sigma_{1}^{2}\right) R_{2}^{2} \\
& \Rightarrow-c=\lambda^{1122}=\lambda^{2233} \\
& \Rightarrow V_{S S}^{(2)}=2 \cdot \frac{1}{8}\left(\lambda^{1122}+\lambda^{2233}\right) F_{S S}(i, j) \\
& =-\frac{c}{2} F_{S S}(i, j)
\end{aligned}
$$


In the case of two real scalar $R_{1}, R_{2}$, we get

$$
\begin{aligned}
\mathcal{L} & =\frac{c}{4} R_{1}^{2} R_{2}^{2} \\
& \Rightarrow-c=\lambda^{1122} \\
& \Rightarrow V_{S S}^{(2)}=2 \cdot \frac{1}{8}\left(\lambda^{1122}\right) F_{S S}(i, j)=-\frac{c}{4} F_{S S}(i, j)
\end{aligned}
$$

and finally, for two equal real scalars $R_{1}=R_{2}$,

$$
\begin{aligned}
\mathcal{L} & =\frac{c}{24} R_{1}^{4} \\
& \Rightarrow-c=\lambda^{1111} \\
& \Rightarrow V_{S S}^{(2)}=\frac{1}{8}\left(\lambda^{1111}\right) F_{S S}(i, i)=-\frac{c}{8} F_{S S}(i, i) .
\end{aligned}
$$

The symmetry factors are summarised in Table 4 .

\section{A.5: SSV}

In C-convention, the interaction between two complex scalars $\phi_{i}, \phi_{j}$ and a complex vector $W_{\mu}^{a}=\left(A_{\mu}^{a}+i B_{\mu}^{a}\right) / \sqrt{2}$ is described by

$\mathcal{L}_{S S V}=c \phi_{i} \overleftrightarrow{\partial^{\mu}} \phi_{j} W_{\mu}^{a}+\mathrm{hc}$

with $c=c(a, i, j)\left(a, i, j\right.$ fixed) and $f \overleftrightarrow{\partial^{\mu}} g=f \partial^{\mu} g-$ $g \partial^{\mu} f$. The same interaction in the R-convention is given by [90, Eq. (2.12)],

$\mathcal{L}_{S S V}=-\sum_{A, I, J} g_{I J}^{A} A_{\mu}^{A} R_{I} \partial^{\mu} R_{J}$

with $g_{I J}^{A}=-g_{J I}^{A}$, real scalars $R_{I}$ and real vectors $A_{\mu}^{A}$. The potential in R-convention is

$V_{S S V}^{(2)}=\frac{1}{4} \sum_{A, I, J}\left(g_{I J}^{A}\right)^{2} F_{S S V}(I, J, A)$

Now break down Eq. (A.164) to real parts,

$$
\begin{aligned}
\mathcal{L}_{S S V}= & c \phi_{i} \overleftrightarrow{\partial^{\mu}} \phi_{j} W_{\mu}^{a}+\mathrm{hc} \\
= & \frac{c}{2 \sqrt{2}}\left(R_{i}+i \sigma_{i}\right) \overleftrightarrow{\partial^{\mu}}\left(R_{j}+i \sigma_{j}\right)\left(A_{\mu}^{a}+i B_{\mu}^{a}\right)+\text { h.c. } \\
= & \frac{\Re(c)}{\sqrt{2}}\left(\left(R_{i} \overleftrightarrow{\partial^{\mu}} R_{j}-\sigma_{i} \overleftrightarrow{\partial^{\mu}} \sigma_{j}\right) A_{\mu}^{a}\right. \\
& \left.-\left(R_{i} \overleftrightarrow{\partial^{\mu}} \sigma_{j}+\sigma_{i} \overleftrightarrow{\partial^{\mu}} R_{j}\right) B_{\mu}^{a}\right) \\
& -\frac{\Im(c)}{\sqrt{2}}\left(\left(\sigma_{i} \overleftrightarrow{\partial^{\mu}} R_{j}+R_{i} \overleftrightarrow{\partial^{\mu}} \sigma_{j}\right) A_{\mu}^{a}+\left(R_{i} \overleftrightarrow{\partial^{\mu}} R_{j}\right.\right. \\
& \left.\left.-\sigma_{i} \overleftrightarrow{\partial^{\mu}} \sigma_{j}\right) B_{\mu}^{a}\right) .
\end{aligned}
$$

There are 4 terms for each $\Re(c)$ and $\Im(c)$ which all involve different fields, thus evaluating Eq. (A.166) gives

$$
\begin{aligned}
V_{S S V}^{(2)}= & 2 \cdot \frac{1}{4}\left(4\left(\frac{\Re(c)}{\sqrt{2}}\right)^{2}+4\left(\frac{\Im(c)}{\sqrt{2}}\right)^{2}\right) \\
& =|c|^{2} F_{S S V}(i, j, a)
\end{aligned}
$$

with a symmetry factor of 2 in front because of $g_{I J}^{A}=-g_{J I}^{A}$. If the two scalars are complex conjugates of each other, $\phi_{j}=$ $\phi_{i}^{*}$, Eq. (A.169) reduces to

$$
\begin{aligned}
\rightarrow & \frac{\Re(c)}{\sqrt{2}}\left(-\left(R_{i} \overleftrightarrow{\partial^{\mu}}\left(-\sigma_{i}\right)+\sigma_{i} \overleftrightarrow{\partial^{\mu}} R_{i}\right) B_{\mu}^{a}\right) \\
& -\frac{\Im(c)}{\sqrt{2}}\left(\left(\sigma_{i} \overleftrightarrow{\partial^{\mu}} R_{i}+R_{i} \overleftrightarrow{\partial^{\mu}}\left(-\sigma_{i}\right)\right) A_{\mu}^{a}\right) \\
= & \frac{2 \Re(c)}{\sqrt{2}}\left(R_{i} \overleftrightarrow{\partial^{\mu}} \sigma_{i} B_{\mu}^{a}\right)-\frac{2 \Im(c)}{\sqrt{2}}\left(\sigma_{i} \overleftrightarrow{\partial^{\mu}} R_{i} A_{\mu}^{a}\right),
\end{aligned}
$$

which gives

$$
\begin{aligned}
V_{S S V}^{(2)}= & 2 \cdot \frac{1}{4}\left(2(\Re(c))^{2}+2(\Im(c))^{2}\right) \\
= & |c|^{2} F_{S S V}(i, j, a) .
\end{aligned}
$$

However, if the vector is real and $\phi_{i}=\phi_{j}^{*}$, Eq. (A.167) becomes

$\mathcal{L}_{S S V}=i c\left(\sigma_{i} \overleftrightarrow{\partial^{\mu}} R_{i}\right) A_{\mu}^{a}+$ h.c.,

where the Hermitean conjugate can be dropped if $c$ is chosen purely imaginary from the start. If that is the case,

$V_{S S V}^{(2)}=\frac{1}{2}|c|^{2} F_{S S V}(i, j, a)$.

If another field is considered real, a factor of $\sqrt{2}$ disappears in the denominator and we end up with half the terms in $\mathcal{L}$, which gives again $V_{S S V}^{(2)}=|c|^{2} F_{S S V}(i, j, a)$. Note that for two real equal scalars, $\mathcal{L}_{S S V}$ vanishes. All the cases are collected in Table 5.

Table 4 Prefactors for $S S$. The contribution is $V_{S S}^{(2)}=k$. $(-c) F_{S S}\left(m_{1}^{2}, m_{2}^{2}\right)$. The table shows $k$ for various cases. The masses of $\phi_{1,2}$ are $m_{1,2}$

\begin{tabular}{lll}
\hline Fields & $\phi_{1,2}$ different & $\phi_{1}=\phi_{2}$ equal \\
\hline$\phi_{1,2} \in \mathbb{C}$ & 1 & $1 / 2$ \\
$\phi_{1} \in \mathbb{C}, \phi_{2} \in \mathbb{R}$ & $1 / 2$ & - \\
$\phi_{1,2} \in \mathbb{R}$ & $1 / 4$ & $1 / 8$ \\
\hline
\end{tabular}

Table 5 This table gives $k$ for the $S S V$ contribution, $V_{S S V}^{(2)}=k$. $|c|^{2} F_{S S V}(i, j, a)$

\begin{tabular}{ll}
\hline Fields & $k$ \\
\hline$\phi_{i}, \phi_{j}, V$ & 1 \\
$\phi_{i}=\phi_{j}^{*}, V \in \mathbb{C}$ & 1 \\
$\phi_{i}=\phi_{j}^{*}, V \in \mathbb{R}$ & $1 / 2$ \\
Else & 1 \\
\hline
\end{tabular}


Example: $\widetilde{q}_{i}^{b}, \widetilde{q}_{i}^{c *}, g^{a}$ with $c=-\frac{g_{3}}{2}\left(\lambda^{a}\right)_{c b}$ and $\lambda^{a}$ the GellMann matrices.

$$
\Rightarrow V_{\widetilde{q}_{i}}^{(2)} \widetilde{q}_{i}^{*} g=\frac{1}{2}\left|\frac{g_{3}}{2}\right|^{2} \underbrace{\left(\sum_{a=1}^{8} \sum_{b, c=1}^{3}\left|\lambda_{b c}^{a}\right|^{2}\right)}_{16} F_{S S V}\left(\widetilde{q}_{i}, \widetilde{q}_{i}, 0\right)
$$

$$
=2 g_{3}^{2} F_{S S V}(\widetilde{q}, \tilde{q}, 0) \quad \text { c.f. [25], (3.48). }
$$

\section{References}

1. CMS Collaboration, S. Chatrchyan et al., Phys. Lett. B 716, 30-61 (2012). arXiv:1207.7235

2. ATLAS Collaboration, G. Aad et al., Phys. Lett. B 716, 1-29 (2012). arXiv:1207.7214

3. H.E. Haber, R. Hempfling, Phys. Rev. Lett. 66, 1815-1818 (1991)

4. J.R. Ellis, G. Ridolfi, F. Zwirner, Phys. Lett. B 257, 83-91 (1991)

5. Y. Okada, M. Yamaguchi, T. Yanagida, Prog. Theor. Phys. 85, 1-6 (1991)

6. Y. Okada, M. Yamaguchi, T. Yanagida, Phys. Lett. B 262, 54-58 (1991)

7. J.R. Ellis, G. Ridolfi, F. Zwirner, Phys. Lett. B 262, 477-484 (1991)

8. A. Brignole, Phys. Lett. B 281, 284-294 (1992)

9. P.H. Chankowski, S. Pokorski, J. Rosiek, Phys. Lett. B 274, 191198 (1992)

10. A. Dabelstein, Z. Phys. C 67, 495-512 (1995). hep-ph/9409375

11. D.M. Pierce, J.A. Bagger, K.T. Matchev, R.-J. Zhang, Nucl. Phys. B 491, 3-67 (1997). hep-ph/9606211

12. R. Hempfling, A.H. Hoang, Phys. Lett. B 331, 99-106 (1994). hep-ph/9401219

13. M.S. Carena, M. Quiros, C. Wagner, Nucl. Phys. B 461, 407-436 (1996). hep-ph/9508343

14. S. Heinemeyer, W. Hollik, G. Weiglein, Phys. Rev. D 58, 091701 (1998). hep-ph/9803277

15. R.-J. Zhang, Phys. Lett. B 447, 89-97 (1999). hep-ph/9808299

16. S. Heinemeyer, W. Hollik, G. Weiglein, Eur. Phys. J. C 9, 343-366 (1999). hep-ph/9812472

17. S. Heinemeyer, W. Hollik, G. Weiglein, Phys. Lett. B 455, 179191 (1999). hep-ph/9903404

18. J.R. Espinosa, R.-J. Zhang, JHEP 0003, 026 (2000). hep-ph/9912236

19. J.R. Espinosa, R.-J. Zhang, Nucl. Phys. B 586, 3-38 (2000). hep-ph/0003246

20. A. Brignole, G. Degrassi, P. Slavich, F. Zwirner, Nucl. Phys. B 631, 195-218 (2002). hep-ph/0112177

21. G. Degrassi, P. Slavich, F. Zwirner, Nucl. Phys. B 611, 403-422 (2001). hep-ph/0105096

22. S.P. Martin, Phys. Rev. D 67, 095012 (2003). hep-ph/0211366

23. A. Brignole, G. Degrassi, P. Slavich, F. Zwirner, Nucl. Phys. B 643, 79-92 (2002). hep-ph/0206101

24. A. Dedes, P. Slavich, Nucl. Phys. B 657, 333-354 (2003). hep-ph/0212132

25. S.P. Martin, Phys. Rev. D 66, 096001 (2002). hep-ph/0206136

26. A. Dedes, G. Degrassi, P. Slavich, Nucl. Phys. B 672, 144-162 (2003). hep-ph/0305127

27. S. Heinemeyer, W. Hollik, H. Rzehak, G. Weiglein, Eur. Phys. J. C 39, 465-481 (2005). hep-ph/0411114
28. K. Sasaki, M.S. Carena, C. Wagner, Nucl. Phys. B 381, 66-86 (1992)

29. M.S. Carena, J. Espinosa, M. Quiros, C. Wagner, Phys. Lett. B 355, 209-221 (1995). hep-ph/9504316

30. H.E. Haber, R. Hempfling, A.H. Hoang, Z. Phys. C 75, 539-554 (1997). hep-ph/9609331

31. M.S. Carena, H. Haber, S. Heinemeyer, W. Hollik, C. Wagner et al., Nucl. Phys. B 580, 29-57 (2000). hep-ph/0001002

32. M.S. Carena, J.R. Ellis, A. Pilaftsis, C. Wagner, Nucl. Phys. B 586, 92-140 (2000). hep-ph/0003180

33. M.S. Carena, J.R. Ellis, A. Pilaftsis, C. Wagner, Nucl. Phys. B 625, 345-371 (2002). hep-ph/0111245

34. J. Espinosa, I. Navarro, Nucl. Phys. B 615, 82-116 (2001). hep-ph/0104047

35. B. Allanach, Comput. Phys. Commun. 143, 305-331 (2002). hep-ph/0104145

36. B. Allanach and M. Bernhardt, Comput. Phys. Commun. 181, 232-245 (2010). arXiv:0903.1805

37. B. Allanach, A. Bednyakov, R. R. de Austri (2014) arXiv: 1407.6130

38. W. Porod, Comput. Phys. Commun. 153, 275-315 (2003). hep-ph/0301101

39. W. Porod, F. Staub, Comput. Phys. Commun. 183, 2458-2469 (2012). arXiv:1104.1573

40. A. Djouadi, J.-L. Kneur, G. Moultaka, Comput. Phys. Commun. 176, 426-455 (2007). hep-ph/0211331

41. S. Heinemeyer, W. Hollik, G. Weiglein, Comput. Phys. Commun. 124, 76-89 (2000). hep-ph/9812320

42. T. Hahn, S. Heinemeyer, W. Hollik, H. Rzehak, G. Weiglein, Comput. Phys. Commun. 180, 1426-1427 (2009)

43. J. Lee, A. Pilaftsis, M.S. Carena, S. Choi, M. Drees et al., Comput. Phys. Commun. 156, 283-317 (2004). hep-ph/0307377

44. J.R. Ellis, J.S. Lee, A. Pilaftsis, Mod. Phys. Lett. A 21, 1405-1422 (2006). hep-ph/0605288

45. S.P. Martin, Phys. Rev. D 75, 055005 (2007). hep-ph/0701051

46. P. Kant, R. Harlander, L. Mihaila, M. Steinhauser, JHEP 1008, 104 (2010). arXiv: 1005.5709

47. R. Harlander, P. Kant, L. Mihaila, M. Steinhauser, Phys. Rev. Lett. 100, 191602 (2008). arXiv:0803.0672

48. S.P. Martin, Phys. Rev. D 71, 016012 (2005). arXiv:hep-ph/0405022

49. S. Borowka, T. Hahn, S. Heinemeyer, G. Heinrich, W. Hollik (2014). arXiv:1404.7074

50. W. Hollik, S. Paßehr (2014). arXiv:1409.1687

51. G. Degrassi, S. Di Vita, P. Slavich (2014). arXiv:1410.3432

52. P. Draper, G. Lee, C. E. M. Wagner, Phys. Rev. D 89, 055023 (2014). arXiv:1312.5743

53. E. Bagnaschi, G. F. Giudice, P. Slavich, A. Strumia (2014). arXiv: 1407.4081

54. G. Degrassi, P. Slavich, Nucl. Phys. B 825, 119-150 (2010). arXiv:0907.4682

55. F. Staub, W. Porod, B. Herrmann, JHEP 1010, 040 (2010). arXiv: 1007.4049

56. K. Ender, T. Graf, M. Muhlleitner, H. Rzehak, Phys. Rev. D 85, 075024 (2012). arXiv: 1111.4952

57. T. Graf, R. Grober, M. Muhlleitner, H. Rzehak, K. Walz, JHEP 1210, 122 (2012). arXiv: 1206.6806

58. U. Ellwanger, C. Hugonie, A. M. Teixeira, Phys. Rep. 496, 1-77 (2010). arXiv:0910.1785

59. U. Ellwanger, C. Hugonie, Mod. Phys. Lett. A 22, 1581-1590 (2007). hep-ph/0612133

60. E. Ma, Phys. Lett. B 705, 320-323 (2011). arXiv:1108.4029

61. Y. Zhang, H. An, X.-D. Ji, R. N. Mohapatra, Phys. Rev. D 78 , 011302 (2008). arXiv:0804.0268

62. M. Hirsch, M. Malinsky, W. Porod, L. Reichert, F. Staub, JHEP 1202, 084 (2012). arXiv: 1110.3037 
63. M. Bastero-Gil, C. Hugonie, S. King, D. Roy, S. Vempati, Phys. Lett. B489, 359-366 (2000). hep-ph/0006198

64. R. Dermisek, J.F. Gunion, Phys. Rev. D73, 111701 (2006). hep-ph/0510322

65. U. Ellwanger, G. Espitalier-Noel, C. Hugonie, JHEP 1109, 105 (2011). arXiv:1107.2472

66. G. G. Ross, K. Schmidt-Hoberg, Nucl. Phys. B 862, 710-719 (2012). arXiv:1108.1284

67. G. G. Ross, K. Schmidt-Hoberg, F. Staub, JHEP 1208, 074 (2012). arXiv: 1205.1509

68. H. K. Dreiner, M. Kramer, J. Tattersall, Europhys. Lett. 99, 61001 (2012). arXiv:1207.1613

69. B. Bhattacherjee, J. L. Evans, M. Ibe, S. Matsumoto, T. T. Yanagida, Phys. Rev. D 87(11), 115002 (2013). arXiv:1301.2336

70. U. Ellwanger, A. M. Teixeira (2014), arXiv:1406.7221

71. J.S. Kim, K. Rolbiecki, K. Sakurai, J. Tattersall 1406, 0858 (2014)

72. F. Staub (2014). arXiv: 1409.7182

73. F. Staub (2008). arXiv:0806.0538

74. F. Staub, Comput. Phys. Commun. 181, 1077-1086 (2010). arXiv:0909.2863

75. F. Staub, Comput. Phys. Commun. 182, 808-833 (2011). arXiv: 1002.0840

76. F. Staub, Comput. Phys. Commun. 184, 1792-1809 (2013). arXiv: 1207.0906

77. F. Staub, Comput. Phys. Commun. 185, 1773-1790 (2014). arXiv: 1309.7223

78. J. Louis, K. Schmidt-Hoberg, L. Zarate (2014). arXiv:1402.2977

79. A. Kaminska, G. G. Ross, K. Schmidt-Hoberg, F. Staub, JHEP 1406, 153 (2014). arXiv: 1401.1816

80. C. Arina, V. Martin-Lozano, G. Nardini (2014). arXiv:1403.6434

81. K. Benakli, M. D. Goodsell, F. Staub, JHEP 1306, 073 (2013). arXiv: 1211.0552

82. K. Benakli, M. Goodsell, F. Staub, W. Porod (2014). arXiv: 1403.5122
83. P. Dießner, J. Kalinowski, W. Kotlarski, D. Stöckinger (2014). arXiv: 1410.4791

84. B. O'Leary, W. Porod, F. Staub, JHEP 1205, 042 (2012). arXiv: 1112.4600

85. M. Hirsch, W. Porod, L. Reichert, F. Staub, Phys. Rev. D 86, 093018 (2012). arXiv:1206.3516

86. G. Brooijmans, R. Contino, B. Fuks, F. Moortgat, P. Richardson, et al. (2014). arXiv:1405.1617

87. S. Banerjee, P. S. B. Dev, S. Mondal, B. Mukhopadhyaya, S. Roy, JHEP 1310, 221 (2013). arXiv:1306.2143

88. A. Bharucha, A. Goudelis, M. McGarrie, Eur. Phys. J. C 74, 2858 (2014). arXiv: 1310.4500

89. P. Athron, J.-H. Park, D. Stöckinger, A. Voigt (2014). arXiv: 1406.2319

90. S.P. Martin, Phys. Rev. D 65, 116003 (2002). arXiv:hep-ph/0111209

91. I. Jack, D.T. Jones, S.P. Martin, M.T. Vaughn, Y. Yamada, Phys. Rev. D 50, 5481-5483 (1994). arXiv:hep-ph/9407291

92. G. Degrassi, S. Heinemeyer, W. Hollik, P. Slavich, G. Weiglein, Eur. Phys. J. C 28, 133-143 (2003). hep-ph/0212020

93. O. Buchmueller, M. Dolan, J. Ellis, T. Hahn, S. Heinemeyer, et al., Eur. Phys. J. C 74, 2809 (2014). arXiv:1312.5233

94. C. Ridders, IEEE Trans. Circuits Syst. 26(11), 979-980 (1979)

95. M. D. Goodsell, K. Nickel, F. Staub, in preparation (2015)

96. S. P. Martin, Phys. Rev. D 89, 013003 (2014). arXiv:1310.7553

97. J. Elias-Miro, J. Espinosa, T. Konstandin, JHEP 1408, 034 (2014). arXiv: 1406.2652

98. S. P. Martin, Phys. Rev. D 90, 016013 (2014). arXiv:1406.2355

99. M. D. Goodsell, K. Nickel, F. Staub. arXiv:1411.4665

100. W. Porod, F. Staub, A. Vicente (2014). arXiv:1405.1434

101. F. Staub, T. Ohl, W. Porod, C. Speckner, Comput. Phys. Commun. 183, 2165-2206 (2012). arXiv: 1109.5147

102. N. Bernal, A. Djouadi, P. Slavich, JHEP 0707, 016 (2007). arXiv:0705.1496 